\title{
Image Clustering: An Unsupervised Approach to Categorize Visual Data in Social Science Research
}

\author{
Han Zhang $^{1}$ and Yilang Peng ${ }^{2}$ \\ ${ }^{1}$ Division of Social Science, Hong Kong University of Science and Technology \\ ${ }^{2}$ Department of Financial Planning, Housing and Consumer Economics at the \\ University of Georgia
}

\begin{abstract}
Automated image analysis has received increasing attention in social scientific research, yet existing scholarship has focused on the application of supervised machine learning to classify images into predefined categories. This study focuses on the task of unsupervised image clustering, which automatically finds categories from image data. First, we review the steps to perform image clustering, and then we focus on the key challenge of performing unsupervised image clustering-finding low-dimensional representations of images. We present several methods of extracting low-dimensional representations of images, including the traditional bag-of-visual-words model, self-supervised learning, and transfer learning. We compare these methods using two datasets containing images related to protests in China (from Sina Weibo, Chinese Twitter) and to climate change(from Instagram). Results show that transfer learning significantly outperforms other methods. The dataset used in the pretrained model critically determines what categories algorithms can discover.
\end{abstract}




\section{Introduction}

Visual information is indispensable for conveying messages. Visual data can take different forms, including historical images, news photographs, television programs, and social media posts, among others. Scholars have found that images can convey information and trigger emotions (e.g., anger), sometimes more powerfully than text (Paivio, 1990; Casas and Williams, 2019)—as the famous saying suggests, "A picture is worth a thousand words." Visual data has become even more abundant in the current social media age. For instance, YouTube and Instagram, two platforms that predominantly circulate visual content, rank first and third on the list of most used social media sites in the United States (Pew Research Center, 2019).

Image data have been frequently used by sociologists to explore meanings, such as inferring protesters' emotions during protests (Corrigall-Brown and Wilkes, 2012; Oleinik, 2015), understanding how media frame abortion issues(Rohlinger and Klein, 2012), or studying terrorists' visual propaganda strategies (Baele et al., 2020). In these studies, researchers rely on content analysis techniques by looking at images piece by piece and summarizing common themes by hand. As cultural sociologists have known for a long time, human reading of images is subject to reproducibility issues even for small datasets (DiMaggio et al., 2013). As the amount of image data will only become more plentiful in the future due to the quick rise of visual-information-based social networking platforms, traditional content analysis approaches are increasingly subject to scalability concerns.

Computer vision, a subfield of computer science that aims to train computers to understand digital imagery, has provided social scientists with tools for computational visual analysis. An emerging line of social science scholarship uses automated image analysis to answer questions relevant to social scientific research. For example, image analysis has been used for detecting partisan media bias in news photographs (Peng, 2018), predicting poverty from satellite images (Jean et al., 2016), and detecting protests and estimating their size from social media images (Zhang and Pan, 2019; Sobolev et al., 2020). The community of computational social science has also provided reviews and tutorials that explain the task of image classification (Casas and Williams, 2017; Joo 
and Steinert-Threlkeld, 2018; Torres and Cantú, 2019; Williams et al., 2020).

Still, the introductory work and prior application of computer vision in social scientific research has predominantly focused on supervised approaches that train computer algorithms to map input images to predetermined categories. Fewer studies in social scientific research have applied unsupervised methods to discover meaningful categories and patterns from images. Unsupervised methods have already been proven useful for large-scale text data. In particular, topic modeling, an unsupervised technique that automatically finds topics from a collection of textual documents, has become quite popular in recent years (Barberá et al., 2019; Roberts et al., 2016; Murashka et al., 2020). With topic modeling, researchers can efficiently summarize textual data and examine the associations between textual messages and various outcomes. For example, researchers have used topic model for revealing the structure of scientific knowledge (Song et al., 2020), testing how political messages influence audience thinking (Roberts et al., 2016), and exploring cultural meanings in large-scale newspaper texts (DiMaggio et al., 2013; DiMaggio, 2015).

In a similar vein, unsupervised methods of image categorization can also be useful for social scientists to reveal hidden themes and topics in visual media. The computer vision community in computer science has already begun to turn their attention to unsupervised image analysis and has proposed several image clustering methods (Dueck and Frey, 2007; Frey and Dueck, 2007; Guérin et al., 2017). A few social scientific studies have also applied image clustering methods, demonstrating the potential of unsupervised methods in discovering meaningful patterns in visual content, such as Instagram images (Hu et al., 2014; Manikonda and De Choudhury, 2017; Peng, 2020). Still, the social scientific community lacks a comprehensive guide on how to perform image clustering on social scientific visual data and the advantages and caveats of different approaches.

To give readers an example of how image clustering can help social scientists analyze visual data, we turn to a dataset called CASM-China (Zhang and Pan, 2019). CASM-China contains over 136,330 protests and 302,506 Weibo images associated with the protests from 2010 to the middle of 2017. ${ }^{1}$ Zhang and Pan (2019) found image data indispensable for identifying offline protests from

\footnotetext{
${ }^{1}$ Zhang and Pan (2019) designed a two-stage deep learning algorithm to detect offline protests based on both text and images in social media posts from Weibo, the Chinese alternative of Twitter.
} 
online social media data because many protesters attempting to mobilize in China avoid posting textual content, which has been shown prone to censorship (King et al., 2013), but rather post images instead; they nevertheless did not study how protesters use images to mobilize in detail. To explore how protesters use images in Chinese social protests, we randomly selected 60 images from the CASM-China dataset and show them in Figure 1. At first sight, these pictures reveal different aspects of protests in China (with people gathering, holding banners or hand-written petition letters). However, we are not sure whether the sampled images reveal other ways protesters use images for mobilization in the full dataset. We are also not clear about the prevalence of different types of ways that people use images. We need a systematic method to formally identify groups of ways protesters use images during Chinese social protests. In Result, we will show how our proposed unsupervised image clustering algorithm can automatically group images in CASMChina into meaningful categories.

[Figure 1 about here.]

This paper introduces unsupervised image clustering that automatically groups images into meaningful categories. We first summarize the steps of unsupervised image clustering: preprocessing, transforming images into low-dimensional representations, clustering, and validation of results. Then we discuss three specific methods of transforming images into low-dimensional representations that efficiently capture the relevant visual information, which has proven to be the key challenge of image clustering. Finally, we test the performance of the three unsupervised image clustering algorithms on two image datasets, including images of protests based on Chinese social media (Sina Weibo, hereafter Weibo) and images related to climate change on Instagram. We conclude with the advantages and limitations of different image clustering techniques.

\section{Steps in Performing Unsupervised Image Clustering}

Previous research in computer vision has proposed a variety of image clustering methods, ranging from using traditional feature extraction algorithms to deep learning models (Dueck and Frey, 
2007; Frey and Dueck, 2007; Guérin et al., 2017). Several social scientific studies have also applied image clustering methods to discover categories in image data. One line of scholarship in human-computer interaction has applied image clustering to social media users' image posts, revealing the prevalence of different content categories in user-generated content (Hu et al., 2014; Manikonda and De Choudhury, 2017). Peng (2020) categorized Instagram posts from politicians and showed that different visual categories received different levels of audience engagement. Torres (2018) examined news photographs of protests and revealed that media outlets of different political affiliations adopt different visual frames. Across these studies, scholars often transform images into simple, low-dimensional representations that efficiently retain relevant information in the images, and then they apply clustering algorithms on these low-dimensional representations (Guérin et al., 2017; Peng, 2020). This paper first reviews the four basic steps in an image clustering task: (1) preprocessing images; (2) extracting low-dimensional representations; (3) clustering; and (4) interpreting and validating clustering results (Figure 2).

[Figure 2 about here.]

\subsection{Preprocessing Images}

In modern computers, digital images are represented as matrices with numeric values. Mathematically, an 8-bit $a \times b$ grayscale image is represented as a matrix $X$ of size $a \times b$, where each cell (known as a pixel) takes values ranging from 0 to $255\left(2^{8}\right)$, with 0 indicating completely black and 255 indicating completely white. The $a \times b$ matrix $X$ can further be transformed to a vector of length $a \cdot b$ by concatenating each row together. In a color image, each pixel is typically represented as a list of values. For example, RGB (Red, Green, and Blue) color space represents each pixel's color as a combination of red, green, and blue colors. An RGB color image is thus stored as a three-dimensional matrix of size $a \times b \times n$, with $a, b$ indicating the size of the image and $n=3$ representing the number of channels in the color space.

A few preprocessing steps might be taken. First, images from online sources often come in a variety of file formats, such as JPEG, PNG, and GIF. Sometimes a PNG file might have a trans- 
parent background and a fourth channel to indicate transparency, whereas an animated GIF might have multiple frames in the file. Researchers may transform images into one common format to facilitate further processing. In addition, images often come in a variety of sizes, and researchers need to resize them to the same dimension. Large images might take up a lot of storage and processing power. $^{2}$ On the other hand, we also do not want to shrink the image size too small (e.g., $20 \times 20$ ) because that will lose information. Resizing image resolution to be between $100 \times 100$ to $300 \times 300$ is the most common practice in the literature. Finally, many image processing methods require the input images to have the same width and height. There are two ways to achieve this: resizing via interpolation or zero-padding (adding columns or rows of all zeros into the pixel matrix of images until they have the same width and height). Hashemi (2019) found that in practice, resizing and zero-padding achieve similar performances. Panel (b) to (c) in Figure 2 visualizes the process to turn an original image into a resized image and its pixel representation.

\subsection{Extracting Low-Dimensional Representations}

The pixel representation of images is usually too complicated for scholars to perform meaningful calculations because the pixel representations of image data are usually high-dimensional (Bühlmann and Van De Geer, 2011). High-dimensional data means that the dimension of the data vectors is on the same scale or may be larger than the sample size. In typical social science regression modeling using survey data, researchers usually have thousands of observations and tens of regressors such that they rarely encounter high-dimensional data. Image data, however, are nearly always high-dimensional. The vector representation of a typical RGB image of $800 \times 600$ pixels has a length of $800 \cdot 600 \cdot 3=1,440,000$, which exceeds the sample size of datasets typically found in social scientific research. The high-dimensional nature of image data yields major challenges for the statistical methods typically used in social science. ${ }^{3}$

\footnotetext{
${ }^{2}$ An image of resolution $500 \times 500$ takes 25 times more space to store than a $100 \times 100$ image. However, any unsupervised clustering algorithm on $500 \times 500$ would not perform 25 times better than its performance on $100 \times 100$ images.

${ }^{3}$ For instance, classical OLS regression cannot be identified if the number of variables exceeds the number of observations.
} 
Moreover, images usually contain redundant information. For instance, background objects, light, as well as colors are all irrelevant if the study goal is to tell to tell whether an image contains a cat or a dog; only the contour of the animal is relevant. Because raw pixel representations are not only high-dimensional but also contain redundant information, nearly every automated image analysis method will first transform the pixel representation of an image into a low-dimensional vector that also preserves key information in the original image. An RGB image of $800 \times 600$ pixels will be transformed into a vector with length ranging from hundreds to thousands, several scales smaller than the original length of 1,440,000. We call this step extracting low-dimensional representation of images throughout this article. Panel (c) to (d) in Figure 2 visualizes the result of turning the pixel representation matrix of the image into a 4096-dimensional vector.

The earliest methods developed to map images into low-dimensional representations keep the basic features of an image, such as contours of objects (referred as edges in the computer vision literature) and corners (intersection of two edges) and remove other nonbasic information; for an overview, see Szeliski (2010). The next generation of methods combine the basic features to further reduce the length of the image vector. ${ }^{4}$ Nevertheless, these traditional approaches have difficulty keeping relevant information about complex objects in their extracted vectors. How these algorithms extract basic features is also predetermined and does not vary by data. Thus, their performance on specific datasets may be suboptimal.

More recent methods of deep learning can extract low-dimensional image representations, which are optimal for subsequent supervised tasks. Deep learning uses multilayer neural networks to predict output (categorical or continuous variables) from input (in this case, images). Mathematically, an input matrix $X$ is mapped to output $Y$ through multiple functions (also called layers) $h_{i}(\cdot), Y=h_{d}\left(h_{d-1}\left(\cdots\left(h_{1}(X)\right)\right)\right.$. Each layer $h_{i}(\cdot)$ is a nonlinear function applied to a linear weighted sum of its inputs; the weights are learned from the input data. The pioneers of deep learning argue that the ability to learn simple yet meaningful representations of raw input is the major reason why deep learning outperforms the traditional methods (LeCun et al., 2015). The

\footnotetext{
${ }^{4}$ For instance, SIFT identifies scale-invariant features from basic features (Lowe, 2004). HOG descriptors use the magnitude and direction of an edge to further reduce the length of an image vector (Dalal and Triggs, 2005).
} 
reason lies in that deep learning learns image representations in a hierarchical fashion (LeCun et al., 2015). Lower-level neural networks (e.g., $h_{1}(\cdot)$ and $h_{2}(\cdot)$ keep basic features such as edges and corners. Upper-level neural networks learn to combine these basic features. The input to the last neural network is $Z=h_{d-1}\left(\cdots\left(h_{1}(X)\right) . Z\right.$ is usually considered the extracted low-dimensional representation of the input images under deep learning.

Due to deep learning's ability to extract concise yet meaningful representations, it achieves superior performance on many supervised tasks. Notably, deep learning-based algorithms significantly outperform algorithms that use traditional approaches in the ImageNet Large Scale Visual Recognition Challenge, a task that classifies 1.28 million images into 1,000 object categories (we refer this dataset as the ImageNet dataset in this article) (Deng et al., 2009). ${ }^{5}$ Three supervised deep learning models are the most widely used in the literature: AlexNet (Krizhevsky et al., 2012), VGGNet (Simonyan and Zisserman, 2015), and ResNet (He et al., 2016). Each has achieved good performance, winning first or second place, in the ImageNet Challege in 2012, 2014 and 2015 respectively. These three models differ in their architecture, including the forms of the $h$ function used and the depth of neural networks $(d)$, among other model details.

Although standard deep learning models outperform traditional methods in their ability to extract a meaningful low-dimensional representation of raw images, they cannot be directly applied to unsupervised image analysis because by definition researchers have input images but do not have their labels yet. In Section 3, we will elaborate on several traditional and deep learning methods that map an image to a low-dimensional vector. Now, we proceed by assuming that images are already transformed into low-dimensional vectors.

\subsection{Clustering}

After low-dimensional vectors are obtained, scholars can apply standard clustering algorithms on them in order to group similar images together. There is a massive literature on clustering algo-

\footnotetext{
${ }^{5}$ The ImageNet project is a large image database that has more than 14 million pictures of 21,841 categories (http://image-net.org/about-stats). The ImageNet project runs the ImageNet Large Scale Visual Recognition Challenge, which uses a subset of the full ImageNet project.
} 
rithms; for a review, see Hastie et al. (2009) and Murphy (2012). Clustering algorithms differ regarding whether a unit (here, an image) can belong to a single category only (single membership) or whether it can be assigned to multiple groups with different belonging probabilities (mixed membership). Single-membership algorithms include k-means, affinity propagation, hierarchical clustering, among others. Mixed-membership clustering algorithms have proven to be especially useful for text and network data (Blei et al., 2003; Airoldi et al., 2009; Roberts et al., 2014).

In practice, we found that extracting low-dimensional representations, rather than the choice of clustering algorithms, is the bottleneck of unsupervised image clustering. If provided with the same low-dimensional image vectors, we found that k-means, hierarchical clustering, and Gaussian Mixture Model (a popular mixed-membership clustering model) yield similar results. Therefore, we use one of the simplest and widely used clustering methods, k-means, in the main analysis, and perform robustness checks of other clustering algorithms in Appendix B.

\subsection{Interpreting and Validating Results}

After the clustering algorithm sorts images into clusters, researchers then interpret the clusters and validate the final solution. Before interpreting the clusters, we first recommend that scholars visualize their clustering solutions. We can sample $M$ images from each of $K$ clusters and place all the images on a $K * M$ canvas. In other words, the collated image looks like Figure 1 except that in the collated image each row represents a cluster. There are two ways to sample images from each cluster for visualization depending on the research purpose:

- Representative sampling. One can select $M$ images that are closest to each cluster center (most representative images) and give a name to the cluster. This approach can help researchers quickly summarize the main theme of each cluster.

- Random sampling. One can also select a random subset of images from each cluster and see whether they belong to the same category. This approach can help researchers validate the clustering solutions by checking if images in a cluster are indeed formulating a coherent 
theme.

Inspecting the collated image helps to rule out some obvious unsatisfactory solutions, but as we will show in Section 4, it may not always easy for researchers to distinguish two subtly different clustering solutions (e.g., choosing between $K=6$ or 8 ). We further propose a method to determine within-cluster consistency to quantitatively measure the extent to which our clustering solutions identify coherent clusters that are internally consistent, which is similar to the so-called "semantic validity" score used to validate the topic modeling results (Quinn et al., 2010; Grimmer and Stewart, 2013). Three steps can be used to calculate within-cluster consistency:

1. Randomly sample $M$ images from each cluster. We used 20 in practice.

2. Instruct research assistants to give each image $i$ a label, $l_{i}$.

3. Calculate the within-cluster consistency for cluster $C_{j}$ as $\alpha_{j}=\frac{\sum_{i=\epsilon C_{j}} \mathbf{I}\left(l_{i}=\operatorname{mode}\left(C_{j}\right)\right)}{\left|C_{j}\right|}$, where $\mathbf{I}$ is an indicator function and mode $\left(C_{j}\right)$ returns the mode of label in $C_{j}$ (i.e., the most common label in cluster $C_{j}$ ). In plain language, the within-cluster consistency $\alpha_{j}$ is the proportion of the images in a given cluster that have the most common label in that cluster.

After calculating the within-cluster consistency of each cluster, we proceed two criteria to rule out unsatisfactory solutions and further choose the best performing ones:

1. First, we need to rule out clustering solutions that have at least one cluster with low withincluster consistency $\alpha_{j}$. A small $\alpha_{j}$, such as $40 \%$, means that even the most common label only characterizes $40 \%$ of the images in that cluster, and the remaining $60 \%$ belong to different label groups. In this case, researchers may need to increase $K$ (the number of clusters) to further separate the cluster into two smaller clusters.

2. Next, we calculate average within-cluster consistency across different clusters for each clustering solution. The higher the average consistency, the more images in that category belong to a common theme, and thus the better the clustering solution. 
In Section 4, we first visually inspect the collated image and then calculate within-cluster consistency for different clustering solutions (different $K$ and different methods). These validations help us finalize our clustering solutions. ${ }^{6}$

\section{Methods for Extracting Low-Dimensional Representations}

Having outlined the steps in image clustering, we now move to the key challenge of unsupervised image analysis: mapping pixel representations of an image to a low-dimensional vector that preserves image information. We introduce one traditional approach and two deep learning-based approaches. Figure 3 illustrates the three approaches.

[Figure 3 about here.]

\subsection{Bag-of-Visual-Words Model}

The bag-of-visual-words model is a widely used traditional method to map an image into a lowdimensional representation (Sivic and Zisserman, 2003; Csurka et al., 2004); it has also caught social scientists' attention (Torres, 2018). The bag-of-visual-words model is based on the SIFT algorithm, which extracts scale-invariant (i.e., invariant to image scaling and rotation) basic features (Lowe, 1999, 2004). The SIFT algorithm, however, will map different images into vectors of different lengths, and thus cannot be directly used for standard clustering algorithms such as kmeans. The bag-of-visual-words model transforms the output of the SIFT algorithm into vectors of the same dimension. It draws inspiration from the famous bag-of-words model in natural language processing (Grimmer and Stewart, 2013). In text analysis, the bag-of-words model represents each document as a vector of occurrence counts of various words; that is, a distribution over the vocabulary. Analogously, the bag-of-visual-word model represents each image as a vector of occurrence

\footnotetext{
${ }^{6}$ It is also popular among practitioners to get a large number of clusters and then refine the results by combining several smaller clusters into a larger category (Hu et al., 2014; Roberts et al., 2014; Manikonda and De Choudhury, 2017; Peng, 2020).To draw an analogy, in topic modeling, scholars can first get a solution with a large number of topics and then classify these topics into a smaller set of theoretically meaningful categories (Song et al., 2020).
} 
counts of image features extracted from SIFT algorithm; that is, a distribution over the "image" vocabulary. $^{7}$

\subsection{Self-Supervised Learning of Image Representations}

As discussed in Section 2.2, standard deep learning models cannot be directly used for unsupervised tasks, where we do not have the labels of images. Self-supervised learning of image features has been recently proposed in deep learning community (Yang et al., 2016; Gidaris et al., 2018; Caron et al., 2018, 2019). ${ }^{8}$ The intuition behind self-supervised learning is that we can perform data augmentation and create pseudo-categories (Gidaris et al., 2018). For instance, we can rotate each image in a dataset by $10^{\circ}, 20^{\circ}, \cdots, 340^{\circ}, 350^{\circ}$, effectively expanding the size of the dataset by 35 . Then a standard supervised deep learning model can be trained that takes the augmented dataset as input and classifies different rotations of the same image into the same group. After this initial step, the last layer can be extracted as the initial low-dimensional vector of images.

We then apply a predetermined clustering algorithm such as k-means on the initial low-dimensional vectors to group them into $K$ clusters. The new cluster assignment is then used as the new pseudocategory, with which we can refit the supervised deep learning model. We iterate between applying a clustering algorithm on the last hidden layer (as low-dimensional vector) to generate new cluster labels as pseudo-category, and using pseudo-category to train supervised deep learning models. This iterative process will continue until some convergence threshold is reached. Upon convergence, we obtain both the low-dimensional vector representation of raw images and its cluster assignment for an unlabelled image dataset. The process is called self-supervised because the entire dataset is used as the training dataset. In contrast, standard supervised learning requires an

\footnotetext{
${ }^{7}$ Technically, the bag-of-visual-words model first concatenates all features produced by the SIFT algorithm, performs clustering on these features, and finds meaningful clusters of SIFT features. These meaningful features are used as the "vocabulary." It then calculates each SIFT vector's occurrence frequency with respect to the vocabulary. The histogram of each SIFT vector over the vocabulary is then used as the final low-dimensional representation of an image. This step is also referred as "codebook generation" in the literature. Further details can be found in standard computer vision textbooks such as (Szeliski, 2010).

${ }^{8}$ Some authors also call this unsupervised learning of image features (Gidaris et al., 2018). We choose to call this branch of research self-supervised learning to distinguish it from our main purpose of the article-unsupervised image clustering.
} 
independent training dataset that has expert-labelled images.

Most studies on self-supervised learning of image representations follow the above iterative process that alternates between training supervised learning algorithms on pseudo-category and then clustering the last layer of the learned models. Their differences lie in the choice of deep learning architecture and clustering algorithms. In practice, we choose the method called "DeepCluster," developed by Caron et al. (2018), because it relies on two widely used deep learning architectures (AlexNet and VGG) and the simplest k-means algorithm for clustering. ${ }^{9}$

\subsection{Transfer Learning}

The second method of mapping an image to a low-dimensional vector is through transfer learning. Transfer learning "borrows information" from existing deep learning models trained on external datasets, typically with millions of images, and repurposes that model to the datasets at hand (Pan and Yang, 2013). The existing deep learning models are often called pretrained models; popular choices of pretrained models include the AlexNet, VGG, and ResNet models trained on the ImageNet Dataset. Technically, to perform transfer learning, researchers feed images into pretrained deep learning models, without changing the model's already learned functional forms and weights. ${ }^{10}$ The last hidden layer is treated as the low-dimensional representation.

Why can we repurpose pretrained models that are trained on a dataset other than scholar's own to obtain low-dimensional representations of images? As we explained earlier, deep learning models map images into low-dimensional vectors in a hierarchical fashion (LeCun et al., 2015). The lower layers in a pretrained model have already "memorized" how to extract basic features from images. Moreover, popular pretrained models, such as those with good performance in ImageNet Challenge, are trained on gigantic datasets with at least millions of images. The sheer data size allows these models to learn how to extract basic features very well.

\footnotetext{
${ }^{9}$ In other work, (Yang et al., 2016) uses a combination of CNN and Recurrent Neural Network (RNN) as the architecture and hierarchical clustering algorithms.

${ }^{10} \mathrm{To}$ draw an analogy with linear regression, someone has fit an OLS regression $y=\beta X$. A different researcher can then use the estimated regression coefficient $\beta$ and new $X$ to predict $Y$.
} 
Where do social scientists find pretrained models? Due to the effectiveness of transfer learning, computer scientists will often release their trained models alongside their publications. Alternatively, popular software for deep learning research (e.g., TensorFlow (Abadi et al., 2015), Keras (Chollet et al., 2015), and PyTorch (Paszke et al., 2019)) contain off-the-shelf, pretrained models based on popular architectures (AlexNet, VGG, and ResNet) and on the ImageNet dataset.

When applying transfer learning, researchers can choose from a myriad of available pretrained models. We highlight three aspects scholars should consider when choosing their pretrained models.

Supervised vs. self-supervised pretrained models. Researchers first must choose between pretrained models based on supervised or self-supervised tasks. Self-supervised pretrained models learn to distinguish images based on their innate differences (e.g., different rotations) (Caron et al., 2018, 2019; Gidaris et al., 2018). On the other hand, supervised models learn a representation of images that are optimal for predicting labels in the training dataset. Even for the same set of images, models trained for predicting one set of categories may not be optimal for predicting another set of categories. For instance, the same images in the ImageNet Challenge dataset can be grouped according to whether they contain the same objects (e.g., cat or tree) or whether their scene differs (e.g, outdoor or indoor scenes). Even using the same ImageNet Dataset, a CNN model trained to detect objects is unlikely to produce image representations suited for detecting scene differences, and vice versa (Krizhevsky et al., 2012; Simonyan and Zisserman, 2015). Therefore, transfer learning using self-supervised pretrained models should provide more general representations. ${ }^{11}$ However, we note that self-supervised learning is still relatively new, while standard supervised deep learning has a long and mature history. Therefore, most off-the-shelf pretrained models are the results of some supervised tasks.

Training dataset of pretrained model. If the researchers' dataset differs significantly from the dataset on which pretrained models are trained, pretrained models will likely to produce less mean-

\footnotetext{
${ }^{11}$ Unless the category used by a supervised pretrained model is very similar to the expected category of images.
} 
ingful presentations. For instance, many popular pretrained models were trained on the ImageNet dataset. If one wants to cluster a dataset full of human faces, a pretrained model (either supervised or self-supervised) trained on ImageNet is unlikely to provide meaningful low-dimensional representations because ImageNet does not contain a person or face class (Zhang and Pan, 2019). In this case, it is better to find a pre-trained model based on human faces, such as the Deep Face Recognition Dataset that contains 2.6 million images and over 2.6 thousand people (Parkhi et al., 2015).

Architecture of pretrained models A more complex network is usually more powerful in learning image representations. For instance, AlexNet has 8 layers, VGG has 16 or 19 layers, and ResNet has 50, 101, or 152 layers. The more complex model performs better on object classification tasks than simple models. However, a more complex architecture is harder to train and is usually slower to work with than a simpler architecture. In practice, we find that VGG often achieves a good balance between its learning power and speed. Scholars who prefer accuracy over speed should also try ResNet.

\section{Performances of Unsupervised Image Clustering Algorithms}

We next designed three studies that use real-world datasets to empirically compare the performance of unsupervised image clustering algorithms. Study 1 compares the performances of the traditional bag-of-visual-words model, self-supervised learning of image representations, and transfer learning. To preview the results, we find that transfer learning significantly outperforms traditional feature extraction methods and self-supervised learning. Study 2 and 3 then focus on transfer learning, testing how the choices of pretrained datasets and architecture impact the clustering results.

Through out this entire section, the results are obtained on a machine with 16 Intel i9-9900K CPU with 3.60GHz clock rate, and 2 GeForce RTX 2080 SUPER GPU. For programming, we used Python, which is the default language for most deep learning research. 


\subsection{Study 1: Comparing Different Methods of Image Clustering}

\subsubsection{Data and Methods}

Our first dataset comes from CASM-China (Zhang and Pan, 2019), as described in the Introduction. From CASM-China, we selected 2,742 offline protest events in the first half of 2016 that have social media images associated with them. ${ }^{12}$ The 2,742 protests contained 14,127 images. We rescaled each image to be of size $224 * 224$ because this is the size of the input used in standard VGG pretrained models.

Method 1 used the bag-of-visual-words model to transform images into low-dimensional features. We expected its performance to be worse than the deep learning-based methods, as described below. Method 2 trained the self-supervised representation learning algorithm (Caron et al., 2018) on the same 14,127 protest Chinese protest images. ${ }^{13}$ Method 3 used transfer learning. We tried two pretrained models. The first used a standard supervised pretrained model using VGG and ImageNet, and the second used self-supervised pretrained model, "DeepCluster" (Caron et al., 2018). ${ }^{14}$

All three methods used K-mean clustering algorithm. K-means requires a critical parameter, the number of clusters $K$. We tried $K$ from each integer from 5 to 15 , and at 20,30, 40, and 50 . We found that $K$ ranging from 5 to 10 yields similar and meaningful results, but $K=6$ yields the highest within-cluster consistency. Below, we visualize the cluster assignment in collated pictures and then present the human validation results with within-cluster consistency, which justifies our choice of $K$.

\subsubsection{Findings}

Figures 4 to 7 are the collated images. Each row represents a cluster identified by the corresponding method. To highlight the main theme of each category, the images in each category were chosen

\footnotetext{
${ }^{12}$ We selected these protests because these predicted events have been validated by research assistants manually.

${ }^{13}$ Technically, we trained 50 epochs using Caron et al. (2018)'s model with VGG architecture..

${ }^{14}$ Both models were trained based on VGG architecture and ImageNet dataset. The first transfer model relies on Keras' native transfer learning model (https://keras.io/api/applications/. The second transfer model was downloaded from the author's website (https://github.com/facebookresearch/deepcluster).
} 
to be a representative sample by selecting those that are closest to the centroid of the cluster.

Method 1 used bag-of-visual-words model (Figure 4). It produced three clusters of images with textual elements (Clusters 3, 4, and 5). Cluster 6 picks up trees and grasses, which is not an theoretically interesting category for understanding contentious politics. Only Cluster 1 and 2 recognize crowd gathering, but still there are wrongly classified pictures (e.g., 1.F and 1.G, which display police violence; 2.B, and 2.J, which display hand-written banners; 2.I, which is a wifi signal irrelevant to protests at all). Considering the fact that we already used images that are the closest to the cluster centroids, such that these images should be the most representative, it suggests that Method 1 does not perform very well.

Method 2, which used self-supervised learning (DeepCluster), also did not produce satisfactory clustering solutions (Figure 5). Although it recognized that trees should not be a separate category, it conflated protester's banners with crowd gathering (Cluster 1), and letters with buildings (Cluster 3). Furthermore, protesters with banners are assigned to different clusters (1, 2, 4, and 5).

Figure 6 and 7 reveal that the transfer learning models (Method 3) both significantly outperformed the two previous methods. Both transfer learning models picked up categories such as gatherings of people (Cluster 1 and 5 in Figure6; Cluster 3 and 5 in Figure 7), hand-written or typed petition letters (Cluster 2 in Figure6; Cluster 6 in Figure 7), screenshots of text (Cluster 3 in Figure 6; Cluster 4 in Figure 7), and protester with banners.

However, transfer learning based on different pretrained models exhibited differences. Transfer learning using supervised pretrained models treated photos with black backgrounds as a separate category. Transfer learning using self-supervised pretrained models recognized that black borders do not have intrinsic meaning; it did not treat photos with black background as a separate class. Moreover, transfer learning using self-supervised pretrained models recognized that photos with protesters holding banners should be put in the same class (Cluster 4 in Figure 7), while transfer learning using supervised pretrained models did not. Overall, transfer learning based on selfsupervised pretrained models produces more meaningful categories. This is expected since selfsupervised learning aims to learn more general features instead of specific features that map images 
to particular categories in the ImageNet Dataset.

The best model — transfer learning based on self-supervised pretrained models-produced theoretically relevant clusters. Cluster 1 in Figure 7 concerns protesters gathering at the gate of government offices; some protesters held banners. Cluster 2, on the other hand, is relevant to protesters blocking streets, which is an especially useful tactic for protesters in China to achieve their goals (Chen, 2009). Cluster 3 are also crowds gathering, with a zoomed-in view. Cluster 4 contains screenshots of text, an approach to avoid censorship on Chinese social media, which heavily relies on detecting texts (King et al., 2013). Cluster 5 is also crowd gathering, but with police presence, which can be potentially used to detect protest policing (della Porta and Reiter, 1998). Cluster 6 contains petition letters, printed or hand-written. Researchers can potentially apply OCR techniques to extract text from letters and infer protesters' goals.

As robustness checks, Figure B1 in Appendix shows results with agglomerative hierarchical clustering and Figure B2 in Appendix shows the results with Gaussian Mixture model; both using self-supervised pre-trained models. Both results are similar to Figure 7, which is obtained by the same low-dimensional vectors but with k-means clustering algorithms.

Another analysis a formal clustering method can achieve, but eyeballing cannot, is to estimate the prevalence of each category in the dataset. Figure 8 plots the number of images in each category. It can be seen that protesters gathering at government offices turn out to be the most prevalent category, and screenshots of texts are the least common category. The prevalence of each category helps researchers to infer the frequency of different mobilization strategies used by Chinese protesters, which has not been well documented.

These results are interesting in two ways. First, the machine-identified categories provide the potential to infer protesters' tactics (e.g., blocking government doors or blocking streets) and goals (from screenshots and texts) from images. Traditionally, this information has often been obtained from text data such as newspapers or social media (Earl et al., 2004; Hutter, 2014). Image data has been suggested as an alternative data source, especially in authoritarian regimes where protesters often use images to avoid platform censorship (Zhang and Pan, 2019). Second, traditionally, when 
researchers study the use of images during protest mobilizations, they mostly examine visual elements such as crowd gathering, fire, and police (Oleinik, 2015; Won et al., 2017; Casas and Williams, 2019). This analysis shows that researchers may overlook protesters' banners and handwritten letters as a type of image used by protesters. It demonstrates the advantage of unsupervised methods to allow researchers to discover patterns in their data that they had not expected beforehand.

[Figure 4 about here.]

[Figure 5 about here.]

[Figure 6 about here.]

[Figure 7 about here.]

[Figure 8 about here.]

\subsubsection{Validation}

Because visual inspection reveals that the traditional bag-of-visual-words model and self-supervised learning does not yield satisfactory performance, we only instructed research assistants to compare the results produced by the two transfer learning models. We present a total of six clustering solutions, which vary by the number of clusters $(6,8$, and 10 clusters), and two transfer learning models, based on supervised and self-supervised pretrained models. For each cluster produced by a pretrained model, we randomly selected 20 images and had research assistants give a label to each image. We then calculated the within-cluster consistency defined earlier.

Figure 9 shows the human validation results. We proceed in two steps. First, we required the minimum threshold of within-cluster consistency to be 0.5 -namely, at least half of the images need to belong to the majority category in that cluster. After this step, only the solutions produced by self-supervised model $(K=6$ or 8$)$ and supervised model $(K=8)$ that met this minimum threshold were kept and other solutions are discarded. Second, we compared the remaining three 
clustering solutions with respect to their average within-cluster consistency. Among the three remaining clusters, the self-supervised model with six clusters produced the highest average withincluster consistency score. Its minimum within-cluster consistency is also significantly larger than the other two remaining solutions (self-supervised model with $K=8$ and supervised model with $K=8$ ). Therefore, we chose the self-supervised model with $K=6$ as the final clustering solution. This choice is the same as our visual inspection in that self-supervised model slightly outperforms the supervised models.

\section{[Figure 9 about here.]}

For a further robustness check, we tried hierarchical clustering as an alternative single-membership clustering algorithm and found that the performances were not different from the results we just presented using k-means as the clustering algorithm (Figure B1 in Appendix). We also tried Gaussian Mixture Model, a standard mixed-membership clustering algorithm that allows images to be assigned to multiple clusters (Figure B2 in Appendix). Surprisingly, we found that only a few images could be assigned to multiple categories, a result we are still investigating now. These results support our argument that the choice of clustering algorithm models are secondary to the choice of methods for learning feature representations.

Overall, study 1 shows that traditional feature extraction methods were less satisfactory. Selfsupervised learning using our own dataset, although seemingly attractive and currently popular in computer science, also did not perform well. One possible reason why self-supervised learning did not perform well is that our dataset is still not large enough, being two to three scales smaller than the training data used by pretrained models $(10 \mathrm{~K}$ vs $1 \mathrm{M}-10 \mathrm{M})$. Moreover, both the bag-ofvisual-words and self-supervised learning models took considerable amounts of time to train. In our practice, it took over 24 hours to train the self-supervised learning model (Method 2$)^{15}$ and over 3 hours to train the bag-of-visual-words model (Method 1). On the contrary, the transfer learning models finished within 5 minutes.

\footnotetext{
${ }^{15}$ Technically, we trained 50 epochs.
} 
Moreover, transfer learning is also easier to implement with respect to coding. Python code for implementing Method 3-using the standard supervised pre-trained model to extract lowdimensional vectors-is available in Appendix A. The code demonstrates that we are able to extract low-dimensional vectors within 50 lines of code. Due to the space restriction, we cannot put all codes in the appendix; we will make them public available. Here, we will disclose that Method 1 and 2 needed significantly more time to implement and could not be finished within 50 lines of code. As traditional approaches and self-supervised models are slow, harder to implement, and do not have good performance, we do not recommend them to social scientists who plan to apply unsupervised image clustering methods at this time.

We also compared transfer learning based on supervised and self-supervised pretrained models and found that self-supervised pretrained models were slightly better than supervised pretrained models in terms of average within-cluster consistency. However, self-supervised learning is still a burgeoning area, and there are fewer off-the-shelf pretrained models. On the other hand, standard software for deep learning, such as Keras, TensorFlow, and PyTorch already have built-in support for using supervised transfer learning models. Therefore, if scholars cannot find pretrained selfsupervised models, we recommend using pretrained supervised models.

\subsection{Study 2: Comparing Different Pretrained Datasets}

Having demonstrated that transfer learning significantly outperforms self-supervised learning from scratch and traditional feature extraction methods, hereafter we focus on transfer learning. Study 2 examines how the choice of pretrained datasets affects clustering results. As discussed in Section 3.3, the categories of images in the pretrained dataset affect the features memorized in the pretrained model and therefore, which features are expected to be extracted for the new data. In transfer learning, it is recommended that the dataset at hand share similarity with the dataset used in the chosen pretrained model.

Our second study focuses on discovering themes from images about climate change on Instagram. Previous research has shown that visual messages about climate change often contain a 
broad range of content themes, including protests, scientific activities, natural landscapes, animals, data visualization, and satellite images, to name a few (O’Neill and Smith, 2014). We selected pretrained models that had been trained with three distinct datasets: ImageNet, Places365, and VGGFace.

The ImageNet dataset contains 1.28 million images of 1,000 object categories. A substantial portion of these categories are related to animals and, in particular, different dog breeds. Still, ImageNet features a diversity of content categories, such as natural scenes (e.g., coral reef, lakeside), places (e.g., cinema, restaurant), foods (e.g., pretzel, cheeseburger), vehicles (e.g., aircraft carrier, speedboat), and everyday objects (e.g., joystick, balloon, envelope, volleyball). As visual messages of climate change often contain a variety of content, features learned from a diverse dataset like ImageNet should be particularly helpful in finding general image categories.

In comparison, the Places 365 dataset contains about 1.8 million images of 365 scene categories, which included a broad range of indoor (e.g., conference center, classroom, legislative chamber, kitchen) and outdoor settings (e.g., street, skyscraper, forest, ocean) (Zhou et al., 2017). As pictures of climate change communication often feature natural landscapes, urban environments, industry, protests, and conferences, this model should help us discover meaningful categories particularly related to scenes and settings.

The VGGFace dataset contains about 2.6 million facial images of over 2.6 thousand people (Parkhi et al., 2015). The features extracted should predominantly pertain to human faces. This pretrained model should be less capable of revealing visual categories relevant to our dataset about climate change than the other two models.

\subsubsection{Data and Methods}

In February 2020, we curated a list of eleven popular Instagram accounts that extensively publish content related to climate change by using the search keywords "climate change" and "global warming" on Instagram. We then retrieved these accounts' posts using a Python library instaloader 
(https://instaloader.github.io/). ${ }^{16}$ We kept images published before January 31, 2020 for analysis $(\mathrm{N}=11,873)$.

To facilitate the comparison among the three datasets, we retrieved three pretrained models using the same model architecture, VGG16, but trained on different datasets: ImageNet (accessible at https://keras.io/api/applications/), Places365 (https://github.com/GKalliatakis/Keras-VGG16-places365), and VGGFace (https://github.com/rcmalli/keras-vggface). We extracted the first dense layer after the convolutional blocks in all three models as our low-dimensional vector of images. ${ }^{17}$ These low-dimensional vectors have 4,096 dimensions. For each model, we performed a principal component analysis and kept the first 200 dimensions for clustering. ${ }^{18}$ We then ran k-means clustering with the number of clusters ranging from 5 to 20 .

\subsubsection{Findings}

We first present the collated images of clustering results to demonstrate how the same deep learning architecture on different pretrained datasets affects the categories machine identified. Figure 10, 11, 12 show results with $K=8$ using ImageNet, Places365, and VGGFace, respectively. We randomly selected 20 images from each cluster for inspection.

The solution using ImageNet revealed a variety of visual categories (Figure 10). Three clusters $(3,5$, and 7$)$ are related to textual messages and graphics, with some variations in design aesthetics. Three clusters (1, 2, and 4) are related to human activities. Cluster 1 captures people, usually several individuals, in outdoor environments, mostly natural scenes or rural areas. These people were also viewed from a distance. Cluster 2, in comparison, features mostly crowds, which are in mostly urban environments such as conferences and streets (protesters). Cluster 4 features closeups or medium shots of people and features only a limited number of individuals. Two other clusters also emerged. Cluster 8 features landscape pictures, usually without people. Cluster

\footnotetext{
${ }^{16}$ These eleven accounts are @climate.change.communication, @climatechangetruth, @climatereality, @climatesavemovement, @climemechange, @cnnclimate, @everydayclimatechange, @ipcc@nasaclimatechange, @noaaclimate, @ thisiszerohour. On average, they had 112477.5 followers (Median =64904) at the time of data collection.

${ }^{17}$ Technically, the fc1 layer in ImageNet, fc1 in Places365, and fc6 in VGGFace.

${ }^{18} \mathrm{We}$ tried to perform k-means on the original 4,096-dimension vectors. The performance is similar, but the speed is significantly slower if we use 4,096 dimensional vectors.
} 
6, however, does seem to show a clear cohesive theme. It contains a combination of still objects, animals, and satellite images, potentially because these pictures share similar visual characteristics. It is possible that some types of images (e.g., animals, satellite images) only comprise a small percentage of the dataset. If we chose a large number of clusters, it is possible that this mixed cluster would break down into smaller categories.

[Figure 10 about here.]

The solution using Places365 showed a pattern different from the solution using ImageNet. Four clusters $(2,4,5$, and 7) are related to textual messages and graphics, with variations in design aesthetics. Interestingly, Cluster 4 contains images featuring dense text on a white background, which has not been found in the ImageNet solution. The crucial difference, though, comes in how this solution clusters pictures of people. While the ImageNet solution distinguishes between pictures of crowds and pictures of several individuals, this Places365 solution mostly groups pictures of people based on settings. Cluster 3 features people in indoor settings, such as conference rooms and lecture halls. Cluster 6 mostly features people in outdoor settings, such as streets. Cluster 1 features pictures of landscapes. Importantly, pictures of satellite images are also mostly categorized into this cluster. This cluster has both pictures featuring people and pictures without people. This could be because the scene categories in Places365 only concern the different types of scene, regardless of the presence of people. Finally, Cluster 8 exclusively features wind farms and frequently used symbols in climate change messages. This is likely because the Places365 dataset has a scene category—wind farms — and this niche category has distinct visual characteristics, so this type of image was rediscovered in our dataset regarding climate change. This pattern was absent in ImageNet and VGGFace solutions.

[Figure 11 about here.]

In comparison, the VGGFace solution performed suboptimally. Not surprisingly, this solution discovered two clusters that were entirely related to human faces (Cluster 3 and 7). However, its 
ability to find other cohesive and meaningful categories was limited. Two clusters (Cluster 1 and 4) predominantly featured images of textual messages and graphics, although these two clusters also mixed in a few other types of images. Cluster 6 featured mostly outdoor scenes, mixing pictures of protests and landscapes. There seemed to be no cohesive themes from the other three clusters (Clusters 2, 5, and 8).

[Figure 12 about here.]

\subsubsection{Validation}

Next, we undertook human validation of the internal consistency of the machine-identified clusters. We present a total of nine clustering solutions, which vary by the number of clusters $(6,8$, and 10 clusters) and three pretrained models (ImageNet, Places365, and VGGFace). For each cluster produced by a pretrained model, we randomly selected 20 images and had research assistants assign a label to each image. We then calculated the percentage of images that belong to the most common theme that describes the cluster.

[Figure 13 about here.]

Figure 13 shows the human validation results. Again, we proceeded by first ruling out clustering solutions whose within-cluster consistency did not reach a minimum threshold (0.5). This exercise discarded VGGFace $(K=6$ and 8$)$ and ImageNet $(k=6)$. For the remaining six clustering solutions, VGGFace $(K=10)$ did not form a clear cluster that had a consistent theme; most of the clusters barely reached 0.5 , and it had the lowest average within-cluster consistency. This observation affirms that our previous argument: when the pretrained dataset is relatively dissimilar from the images to be analyzed(as a dataset of faces, such as VGGFace, is dissimilar to climate change images), it is unlikely that the extracted features from the pretrained model can be clustered into coherent themes. The pretrained model based on Places365 dataset $(K=8)$ yielded the highest average within-cluster consistency. Its minimum within-cluster consistency is also the largest 
among all clustering solutions. Therefore, we chose the pretrained model based on the Places365 dataset $(K=8)$ as the final clustering solution.

To conclude, Study 2 reveals the choice of pretrained datasets can significantly shape the categories found in the clustering solution. Among the three solutions, the solution using VGGFace had the worst performance. It was only able to discover categories related to human faces and missed other visual themes. Both the solution using ImageNet and the solution using Places365 were able to find more meaningful categories, yet they differed on the specific emphasis and categories in the solutions.

\subsection{Study 3: Comparing Different Architecture}

Finally, we compared pretrained models trained on the same dataset but using different model architecture. As discussed in Section 3.3, complex models have better ability to extract meaningful low-dimensional representations and thus lead to better clustering results. We next see whether this logical reasoning is correct. Study 3 uses the same dataset, CASM-China, from Study 1.

\subsubsection{Findings}

We applied a pretrained self-supervised learning model. The results are shown in Figure 14. The only difference between Figure 14 and Figure 7 is that the former relies on AlexNet architecture and the latter relies on VGG architecture. Comparing Figure 7 and Figure 14 thus reveals how VGG and AlexNet differ in their ability to find meaningful low-dimensional representations for clustering. Because VGG is more complex in its architecture, in theory, we should expect it to perform better at clustering images.

[Figure 14 about here.]

Indeed, Figure 14 shows that the AlexNet architecture, the simpler architecture, confuses some images. For instance, Cluster 4 conflates screenshots of texts with pictures of petition letters. 
Cluster 6 also conflates crowd gatherings in front of buildings and on roads. The simpler pretrained model indeed produces an inferior clustering solution than a more complex pretrained model.

Overall, we found that using the same pretrained dataset and model, VGG performs well in finding meaningful representations, but AlexNet's performance is not good enough.

\section{Discussion}

This study introduced steps for the task of unsupervised image clustering. We argued that the key challenge is to find concise but meaningful low-dimensional representations of images. We then focused on three methods of finding low-dimensional representations of images: bag-of-visualwords, self-supervised learning, and transfer learning. We evaluated the three methods on two datasets containing images about protests on Weibo and images about climate change on Instagram. We found that transfer learning significantly outperforms the other two methods, not only with respect to clustering performance but also in speed of calculation and ease in coding im-

plementation (Study 1). We further discussed and demonstrated some practical considerations in using transfer models for unsupervised image clustering. We found that the particular pretrained dataset used in transfer learning critically determines the clustering results (Study 2), and a more complex pretrained model in transfer learning generally works better than a simpler counterpart (Study 3).

This article contributes to the burgeoning study of "image as data" by synthesizing practical steps and methods of unsupervised image clustering for social scientists. Image data are abundant in the current social media age, but so far, the analysis of image data in sociology still heavily relies on researchers looking at images and summarizing common themes through traditional content analysis techniques (Krippendorff, 2004; Corrigall-Brown and Wilkes, 2012; Rohlinger and Klein, 2012; Oleinik, 2015). Human reading of images is subject to reproducibility issues even for small datasets, and to scalability concerns for larger image datasets. Much like the advance of text analysis from traditional content analysis to automated, unsupervised methods (especially 
topic modeling) has fostered a wide range of scholarship capturing meaning in large-scale texts (Grimmer and Stewart, 2013; DiMaggio et al., 2013; Wilkerson and Casas, 2017), we believe our proposed method also will help future social scientists capture theoretically interesting information in visual data, reduce its complexity, and provide interpretations.

Unsupervised image clustering does not replace human's reading of images, however. Rather, it provides social scientists a lens to quickly capture meaningful categories from large-scale image datasets and develop these meaningful categories, which can then be used for further theorizing and empirical work. Importantly, we proposed concrete steps (visual inspection through collated images and human coding for within-cluster consistency) to interpret and validate the clustering results. Our article thus strengthens the reproducibility of image analysis by pushing researchers to validate their clustering results instead of offering a post hoc justification for their choice of themes in an image dataset.

It is important to consider the potential biases and ethical implications of computer vision algorithms. Prior research has suggested that machine learning models might incorporate gender, racial, and cultural biases (Zou and Schiebinger, 2018). For example, research has shown that facial recognition models often show higher error rates for gender or racial minorities (Grother et al., 2019; Zou and Schiebinger, 2018). Furthermore, many computer vision models have been trained on datasets that predominately contain visual data from Western contexts. For example, images in the ImageNet dataset used in this study, a dataset popular among computer vision researchers, come from a limited number of cultures, with nearly half of images from the United States (Zou and Schiebinger, 2018). In our results, the pretrained models using ImageNet showed satisfactory results for datasets both from Weibo, a Chinese platform and Instagram. Nevertheless, biases in pretrained models may pose a challenge if researchers are interested in more culture-specific content themes that might not be well reflected in the features learned in pretrained models.

We close by noting limitations and future directions for research. Although we recommend the use of transfer learning for its effectiveness, speed, and relative simplicity to implement, researchers are constrained by the availability of pretrained models. If researchers cannot find pre- 
trained models that are trained on datasets that are similar to their own, then they have to make a compromise of using a less satisfactory pretrained model. Second, in transfer learning for supervised tasks, scholars often adapt pretrained models to their smaller dataset by keeping some learned parameters and weights constant but allowing others to change with their dataset, a process called "fine-tuning" (Zhang and Pan, 2019). However, there has been no standard procedure in fine-tuning pretrained models for unsupervised tasks, which is an opportunity for future methodological research.

We included the code to implement transfer learning using VGGNet and ImageNet dataset in Appendix A. Other codes and data to replicate the analyses in the paper will made publicly available. There are already many good tutorials on transfer learning techniques both inside and outside social science (Williams et al., 2020; Sarkar et al., 2018). It is most common to use Python language to perform transfer learning with pretrained models, but researchers have started to make it possible to use $\mathrm{R}$ to perform transfer learning, too (Ramasubramanian and Singh, 2019). We understand that image analysis is relatively new to social scientists, and so we plan to develop software packages (potentially in R) and operation manuals that make it easier for social scientists to use transfer learning models. 


\section{References}

Abadi, Martín, Ashish Agarwal, Paul Barham, Eugene Brevdo, Zhifeng Chen, Craig Citro, Greg S. Corrado, Andy Davis, Jeffrey Dean, Matthieu Devin, Sanjay Ghemawat, Ian Goodfellow, Andrew Harp, Geoffrey Irving, Michael Isard, Yangqing Jia, Rafal Jozefowicz, Lukasz Kaiser, Manjunath Kudlur, Josh Levenberg, Dan Mané, Rajat Monga, Sherry Moore, Derek Murray, Chris Olah, Mike Schuster, Jonathon Shlens, Benoit Steiner, Ilya Sutskever, Kunal Talwar, Paul Tucker, Vincent Vanhoucke, Vijay Vasudevan, Fernanda Viégas, Oriol Vinyals, Pete Warden, Martin Wattenberg, Martin Wicke, Yuan Yu, and Xiaoqiang Zheng. 2015. "TensorFlow: LargeScale Machine Learning on Heterogeneous Systems.” Software available from tensorflow.org.

Airoldi, Edoardo M, David M. Blei, Stephen E. Fienberg, and Eric P. Xing. 2009. "Mixed Membership Stochastic Blockmodels." In Advances in Neural Information Processing Systems 21, edited by D. Koller, D. Schuurmans, Y. Bengio, and L. Bottou, pp. 33-40. Curran Associates, Inc.

Baele, Stephane J, Katharine A Boyd, and Travis G Coan. 2020. "Lethal Images: Analyzing Extremist Visual Propaganda from ISIS and Beyond.” Journal of Global Security Studies 5:634657.

Barberá, Pablo, Andreu Casas, Jonathan Nagler, Patrick J. Egan, Richard Bonneau, John T. Jost, and Joshua A. Tucker. 2019. "Who Leads? Who Follows? Measuring Issue Attention and Agenda Setting by Legislators and the Mass Public Using Social Media Data." American Political Science Review 113:883-901.

Blei, David M., Andrew Y. Ng, and Michael I. Jordan. 2003. "Latent Dirichlet Allocation.” J. Mach. Learn. Res. 3:993-1022.

Bühlmann, Peter and Sara Van De Geer. 2011. Statistics for high-dimensional data: methods, theory and applications. Springer Science \& Business Media. 
Caron, Mathilde, Piotr Bojanowski, Armand Joulin, and Matthijs Douze. 2018. "Deep Clustering for Unsupervised Learning of Visual Features." In Computer Vision - ECCV 2018, edited by Vittorio Ferrari, Martial Hebert, Cristian Sminchisescu, and Yair Weiss, Lecture Notes in Computer Science, pp. 139-156, Cham. Springer International Publishing.

Caron, Mathilde, Piotr Bojanowski, Julien Mairal, and Armand Joulin. 2019. "Unsupervised PreTraining of Image Features on Non-Curated Data." arXiv:1905.01278 [cs] .

Casas, Andreu and Nora Webb Williams. 2017. "Images That Matter: Online Protests and the Mobilizing Role of Pictures.” SSRN Scholarly Paper ID 2832805, Social Science Research Network, Rochester, NY.

Casas, Andreu and Nora Webb Williams. 2019. "Images That Matter: Online Protests and the Mobilizing Role of Pictures.” Political Research Quarterly 72:360-375.

Chen, Xi. 2009. "The Power of "Troublemaking": Protest Tactics and Their Efficacy in China." Comparative Politics 41:451-471.

Chollet, François et al. 2015. "Keras.” https://keras.io.

Corrigall-Brown, Catherine and Rima Wilkes. 2012. "Picturing Protest: The Visual Framing of Collective Action by First Nations in Canada." American Behavioral Scientist 56:223-243.

Csurka, Gabriella, Christopher R. Dance, Lixin Fan, Jutta Willamowski, and Cédric Bray. 2004. "Visual Categorization with Bags of Keypoints." In In Workshop on Statistical Learning in Computer Vision, ECCV, pp. 1-22.

Dalal, Navneet and Bill Triggs. 2005. "Histograms of Oriented Gradients for Human Detection." In Computer Vision and Pattern Recognition, 2005. CVPR 2005. IEEE Computer Society Conference On, volume 1, pp. 886-893. IEEE.

della Porta, Donatella and Herbert Reiter. 1998. Policing Protest: The Control of Mass Demonstrations in Western Democracies. U of Minnesota Press. 
Deng, Jia, Wei Dong, Richard Socher, Li-jia Li, Kai Li, and Li Fei-fei. 2009. "Imagenet: A Large-Scale Hierarchical Image Database.” In In CVPR.

DiMaggio, Paul. 2015. “Adapting Computational Text Analysis to Social Science (and Vice Versa).” Big Data \& Society 2:2053951715602908.

DiMaggio, Paul, Manish Nag, and David Blei. 2013. "Exploiting Affinities Between Topic Modeling and the Sociological Perspective on Culture: Application to Newspaper Coverage of Us Government Arts Funding." Poetics 41:570-606.

Dueck, Delbert and Brendan J Frey. 2007. "Non-metric affinity propagation for unsupervised image categorization." In 2007 IEEE 11th International Conference on Computer Vision, pp. 1-8. IEEE.

Earl, Jennifer, Andrew Martin, John D. McCarthy, and Sarah A. Soule. 2004. "The Use of Newspaper Data in the Study of Collective Action." Annual Review of Sociology 30:65-80.

Frey, Brendan J and Delbert Dueck. 2007. "Clustering by passing messages between data points." science 315:972-976.

Gidaris, Spyros, Praveer Singh, and Nikos Komodakis. 2018. "Unsupervised Representation Learning by Predicting Image Rotations." In International Conference on Learning Representations.

Grimmer, Justin and Brandon M. Stewart. 2013. "Text as Data: The Promise and Pitfalls of Automatic Content Analysis Methods for Political Texts.” Political Analysis 21:267-297.

Grother, Patrick, Mei Ngan, and Kayee Hanaoka. 2019. Face Recognition Vendor Test (FVRT): Part 3, Demographic Effects. National Institute of Standards and Technology.

Guérin, Joris, Olivier Gibaru, Stéphane Thiery, and Eric Nyiri. 2017. "CNN features are also great at unsupervised classification." arXiv preprint arXiv:1707.01700 . 
Hashemi, Mahdi. 2019. "Enlarging Smaller Images before Inputting into Convolutional Neural Network: Zero-Padding vs. Interpolation.” Journal of Big Data 6:98.

Hastie, Trevor, Robert Tibshirani, Jerome Friedman, T. Hastie, J. Friedman, and R. Tibshirani. 2009. The Elements of Statistical Learning, volume 2. Springer.

He, K., X. Zhang, S. Ren, and J. Sun. 2016. “Deep Residual Learning for Image Recognition.” In 2016 IEEE Conference on Computer Vision and Pattern Recognition (CVPR), pp. 770-778.

Hu, Yuheng, Lydia Manikonda, Subbarao Kambhampati, et al. 2014. "What we instagram: A first analysis of instagram photo content and user types.” In Icwsm.

Hutter, Swen. 2014. "Protest Event Analysis and Its Offspring." In Methodological Practices in Social Movement Research, edited by Donatella Della Porta, pp. 335-367. Oxford University Press.

Jean, Neal, Marshall Burke, Michael Xie, W Matthew Davis, David B Lobell, and Stefano Ermon. 2016. “Combining satellite imagery and machine learning to predict poverty.” Science 353:790_ 794.

Joo, Jungseock and Zachary C. Steinert-Threlkeld. 2018. "Image as Data: Automated Visual Content Analysis for Political Science.” arXiv:1810.01544 [cs, stat] .

King, Gary, Jennifer Pan, and Margaret E. Roberts. 2013. "How Censorship in China Allows Government Criticism but Silences Collective Expression.” American Political Science Review 107:326-343.

Krippendorff, Klaus. 2004. Content Analysis: An Introduction to Its Methodology. SAGE Publications.

Krizhevsky, Alex, Ilya Sutskever, and Geoffrey E Hinton. 2012. "ImageNet Classification with Deep Convolutional Neural Networks." In Advances in Neural Information Processing Systems 
25, edited by F. Pereira, C. J. C. Burges, L. Bottou, and K. Q. Weinberger, pp. 1097-1105. Curran Associates, Inc.

LeCun, Yann, Yoshua Bengio, and Geoffrey Hinton. 2015. “Deep Learning.” Nature 521:436-444.

Lowe, D. G. 1999. “Object Recognition from Local Scale-Invariant Features.” In Proceedings of the Seventh IEEE International Conference on Computer Vision, volume 2, pp. 1150-1157 vol.2.

Lowe, David G. 2004. “Distinctive Image Features from Scale-Invariant Keypoints.” International Journal of Computer Vision 60:91-110.

Manikonda, Lydia and Munmun De Choudhury. 2017. "Modeling and understanding visual attributes of mental health disclosures in social media." In Proceedings of the 2017 CHI Conference on Human Factors in Computing Systems, pp. 170-181.

Murashka, Volha, Jiaying Liu, and Yilang Peng. 2020. "Fitspiration on Instagram: Identifying Topic Clusters in User Comments to Posts with Objectification Features." Health Communication pp. 1-12.

Murphy, Kevin P. 2012. Machine Learning: A Probabilistic Perspective. The MIT Press.

Oleinik, Anton. 2015. "On Content Analysis of Images of Mass Protests: A Case of Data Triangulation.” Quality \& Quantity 49:2203-2220.

O’Neill, Saffron J and Nicholas Smith. 2014. "Climate change and visual imagery." Wiley Interdisciplinary Reviews: Climate Change 5:73-87.

Paivio, Allan. 1990. Mental Representations: A Dual Coding Approach. Oxford University Press.

Pan, Weike and Qiang Yang. 2013. “Transfer Learning in Heterogeneous Collaborative Filtering Domains." Artificial Intelligence 197:39-55. 
Parkhi, Omkar M., Andrea Vedaldi, and Andrew Zisserman. 2015. “Deep Face Recognition.” In Procedings of the British Machine Vision Conference 2015, pp. 41.1-41.12, Swansea. British Machine Vision Association.

Paszke, Adam, Sam Gross, Francisco Massa, Adam Lerer, James Bradbury, Gregory Chanan, Trevor Killeen, Zeming Lin, Natalia Gimelshein, Luca Antiga, Alban Desmaison, Andreas Kopf, Edward Yang, Zachary DeVito, Martin Raison, Alykhan Tejani, Sasank Chilamkurthy, Benoit Steiner, Lu Fang, Junjie Bai, and Soumith Chintala. 2019. "PyTorch: An Imperative Style, High-Performance Deep Learning Library." In Advances in Neural Information Processing Systems 32, edited by H. Wallach, H. Larochelle, A. Beygelzimer, F. d. Alché-Buc, E. Fox, and R. Garnett, pp. 8024-8035. Curran Associates, Inc.

Peng, Yilang. 2018. “Same Candidates, Different Faces: Uncovering Media Bias in Visual Portrayals of Presidential Candidates with Computer Vision." Journal of Communication 68:920-941.

Peng, Yilang. 2020. “What Makes Politicians’ Instagram Posts Popular? Analyzing Social Media Strategies of Candidates and Office Holders with Computer Vision.” The International Journal of Press/Politics p. 1940161220964769.

Pew Research Center. 2019. "Social media fact sheet."

Quinn, Kevin M., Burt L. Monroe, Michael Colaresi, Michael H. Crespin, and Dragomir R. Radev. 2010. "How to Analyze Political Attention with Minimal Assumptions and Costs." American Journal of Political Science 54:209-228.

Ramasubramanian, Karthik and Abhishek Singh. 2019. "Deep Learning Using Keras and TensorFlow." In Machine Learning Using R: With Time Series and Industry-Based Use Cases in R, edited by Karthik Ramasubramanian and Abhishek Singh, pp. 667-688. Berkeley, CA: Apress.

Roberts, Margaret E., Brandon M. Stewart, and Edoardo M. Airoldi. 2014. "Structural Topic Models.” Technical report, Working Paper. Export BibTex Tagged XML. 
Roberts, Margaret E, Brandon M Stewart, and Edoardo M Airoldi. 2016. "A model of text for experimentation in the social sciences." Journal of the American Statistical Association 111:9881003.

Rohlinger, Deana A. and Jesse Klein. 2012. "Visual Landscapes and the Abortion Issue." American Behavioral Scientist 56:172-188.

Sarkar, Dipanjan, Raghav Bali, and Tamoghna Ghosh. 2018. Hands-On Transfer Learning with Python: Implement Advanced Deep Learning and Neural Network Models Using TensorFlow and Keras. Packt Publishing.

Simonyan, Karen and Andrew Zisserman. 2015. "Very Deep Convolutional Networks for LargeScale Image Recognition." In Proceedings of the Third International Conference on Learning Representations.

Sivic and Zisserman. 2003. "Video Google: A Text Retrieval Approach to Object Matching in Videos." In Proceedings Ninth IEEE International Conference on Computer Vision, pp. 1470_ 1477 vol.2.

Sobolev, Anton, M. Keith Chen, Jungseock Joo, and Zachary C. Steinert-Threlkeld. 2020. "News and Geolocated Social Media Accurately Measure Protest Size Variation.” American Political Science Review pp. 1-9.

Song, Hyunjin, Jakob-Moritz Eberl, and Olga Eisele. 2020. "Less Fragmented Than We Thought? Toward Clarification of a Subdisciplinary Linkage in Communication Science, 2010-2019." Journal of Communication 70:310-334.

Szeliski, Richard. 2010. Computer Vision: Algorithms and Applications. Springer Science \& Business Media.

Torres, Michelle. 2018. "Framing a protest: Determinants and effects of visual frames.” Technical report, Working Paper. 
Torres, Michelle and Francisco Cantú. 2019. "Learning to See: Convolutional Neural Networks for the Analysis of Social Science Data."

Wilkerson, John and Andreu Casas. 2017. "Large-Scale Computerized Text Analysis in Political Science: Opportunities and Challenges.” Annual Review of Political Science 20:529-544.

Williams, Nora Webb, Andreu Casas, and John D. Wilkerson. 2020. Images as Data for Social Science Research: An Introduction to Convolutional Neural Nets for Image Classification. Cambridge University Press.

Won, Donghyeon, Zachary C. Steinert-Threlkeld, and Jungseock Joo. 2017. "Protest Activity Detection and Perceived Violence Estimation from Social Media Images." In Proceedings of the 2017 ACM on Multimedia Conference, MM '17, pp. 786-794, New York, NY, USA. ACM.

Yang, Jianwei, Devi Parikh, and Dhruv Batra. 2016. "Joint Unsupervised Learning of Deep Representations and Image Clusters." In 2016 IEEE Conference on Computer Vision and Pattern Recognition (CVPR), pp. 5147-5156.

Zhang, Han and Jennifer Pan. 2019. “CASM: A Deep-Learning Approach for Identifying Collective Action Events with Text and Image Data from Social Media.” Sociological Methodology 49:1-57.

Zhou, Bolei, Agata Lapedriza, Aditya Khosla, Aude Oliva, and Antonio Torralba. 2017. "Places: A 10 million image database for scene recognition." IEEE transactions on pattern analysis and machine intelligence 40:1452-1464.

Zou, James and Londa Schiebinger. 2018. "AI can be sexist and racist—it's time to make it fair." 


\section{List of Figures}

1 Randomly selected images from the CASM-China protest dataset. . . . . . . . . . 39

2 (a) Steps in image clustering. (b-d) An example of feature extraction: (b) the original image $(600 \times 400 \times 3)$; (c) the resized image $(224 \times 224 \times 3)$; (d) the lowdimensional representation $(4096 \times 1)$ using feature extraction with transfer learning. 40

3 Three approaches of learning image representations and clustering. . . . . . . . . . 41

4 Method 1, Bag-of-Visual-Words model. . . . . . . . . . . . . . . . . . 42

5 Method 2, self-supervised learning of image representations (Caron et al., 2018). . 43

6 Method 3, transfer learning using supervised pretrained models. . . . . . . . . . . 44

7 Method 3, transfer learning using self-supervised pretrained models. . . . . . . . . 45

8 Number of images in each class. . . . . . . . . . . . . . . . . . . . . . 46

9 Percentage of images in each cluster that belong to the most common theme in that cluster, for Chinese Protest Dataset. Average percentage $(M)$ is highlighted in red. Six clustering solutions are shown, varying by the number of clusters and the pretrained models. . . . . . . . . . . . . . . . . . . . . . . . 47

10 Eight-cluster solution based on features extracted from a pretrained model trained on ImageNet dataset. . . . . . . . . . . . . . . . . . . . . . 48

11 Eight-cluster solution based on features extracted from a pretrained model trained on the Places365 dataset. . . . . . . . . . . . . . . . . . . . . . . . . . 49

12 Eight-cluster solution based on features extracted from a pretrained model trained on the VGGFace dataset. . . . . . . . . . . . . . . . . . . . . . . . 50

13 Percentage of images that belong to the most common theme in each cluster, for climate change dataset. Average percentage $(M)$ is highlighted in red. Nine clustering solutions are shown, varying by the number of clusters and pretrained models. 51

14 Method 3, transfer learning using self-supervised pretrained models (AlexNet as the architecture), on Protest Dataset . . . . . . . . . . . . . . . . 52

B1 Transfer learning using self-supervised model, using agglomerative hierarchical clustering. Results are similar to Figure 7 where other things are equal but clustering algorithm is k-means. . . . . . . . . . . . . . . . . .

B2 Transfer learning using self-supervised model, using Gaussian Mixture Model. Results are similar to Figure 7 where other things are equal but clustering algorithm

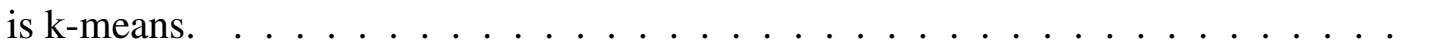



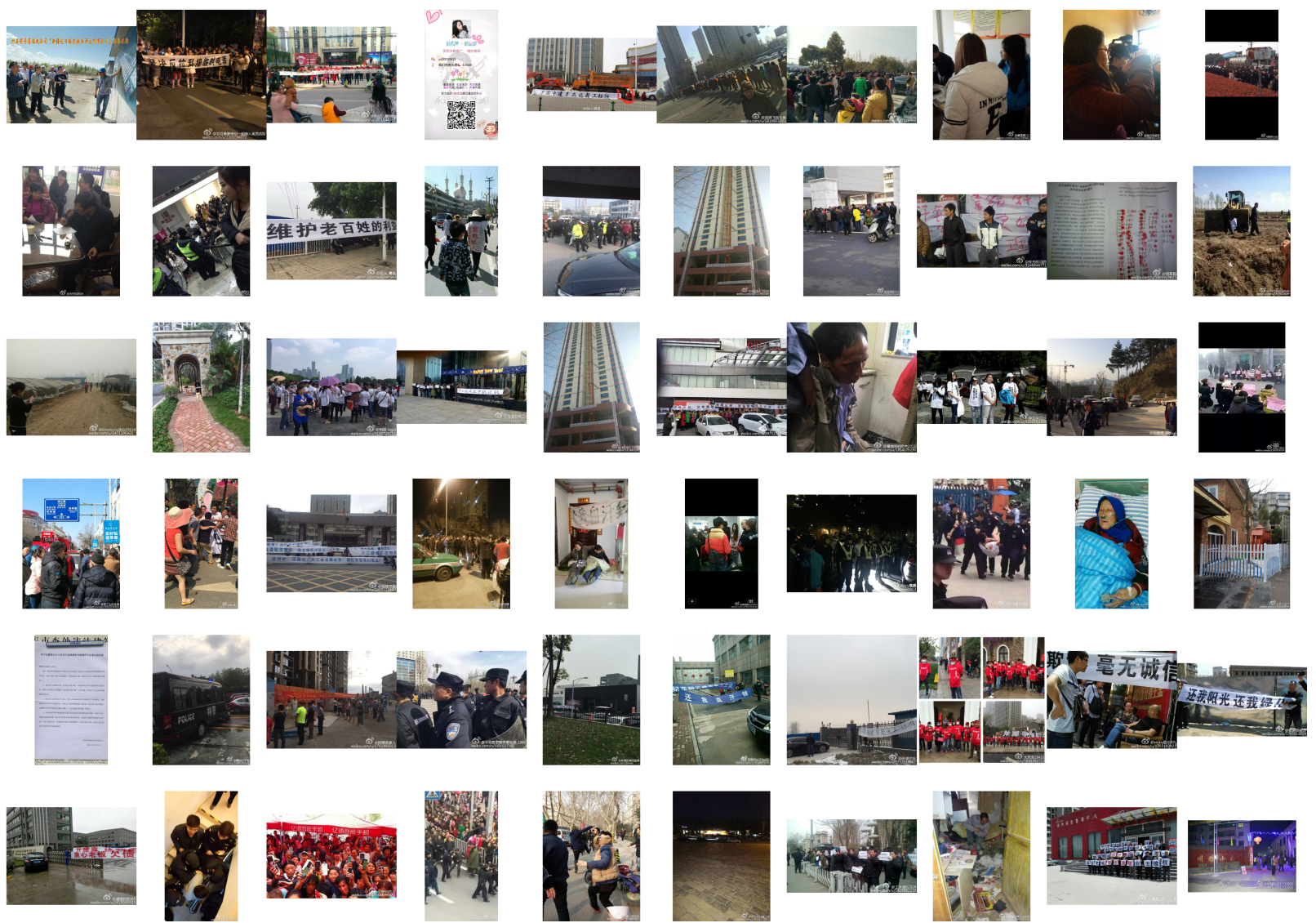

Figure 1: Randomly selected images from the CASM-China protest dataset. 
a

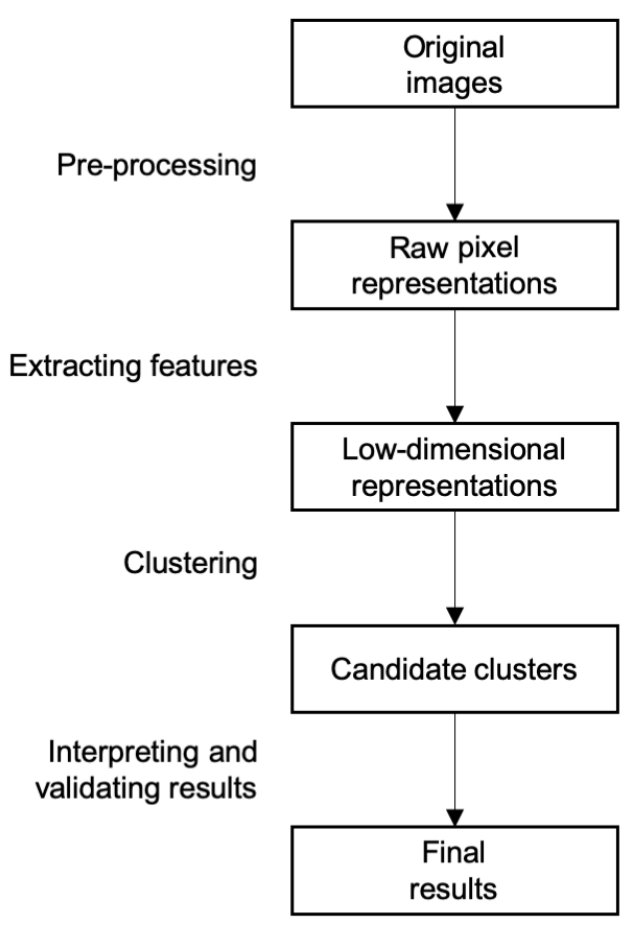

b

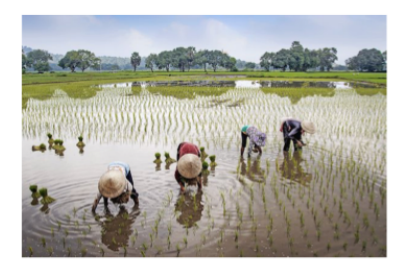

C

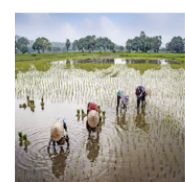

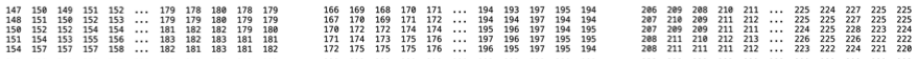

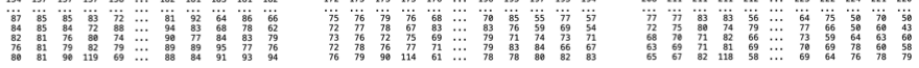

d

Figure 2: (a) Steps in image clustering. (b-d) An example of feature extraction: (b) the original image $(600 \times 400 \times 3)$; (c) the resized image $(224 \times 224 \times 3)$; (d) the low-dimensional representation $(4096 \times 1)$ using feature extraction with transfer learning. 
(a) Bag of visual words

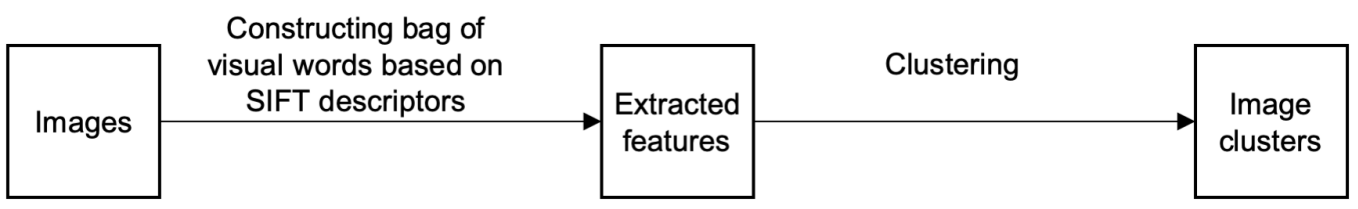

(b) Self-supervised learning

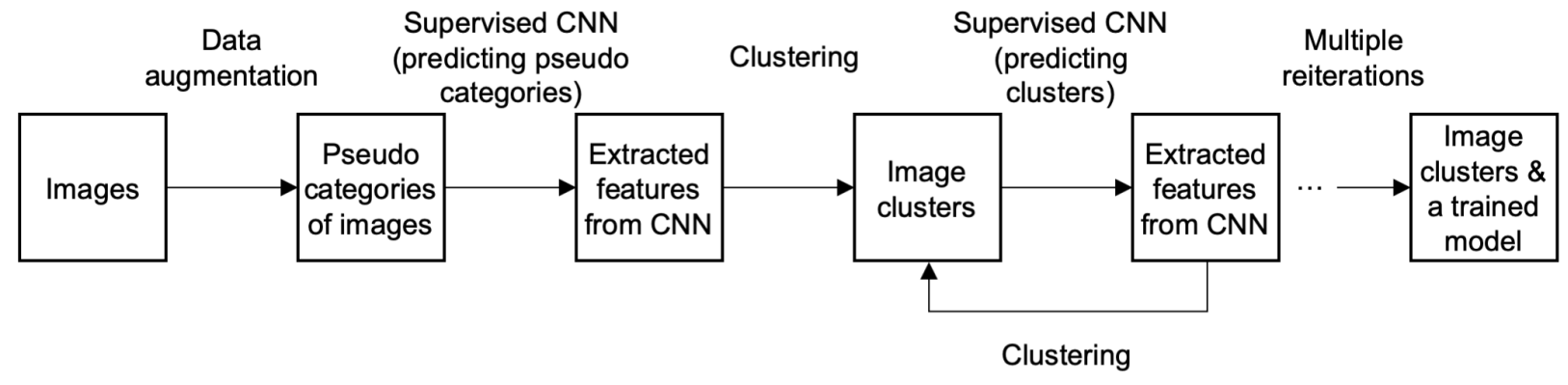

(c) Transfer learning

\begin{tabular}{|l|c|c|c|}
\multirow{2}{*}{ Images } & $\begin{array}{c}\text { Feature extraction with } \\
\text { a pre-trained model }\end{array}$ & $\begin{array}{c}\text { Extracted } \\
\text { features }\end{array}$ & Clustering \\
\cline { 3 - 3 }
\end{tabular}

Figure 3: Three approaches of learning image representations and clustering. 

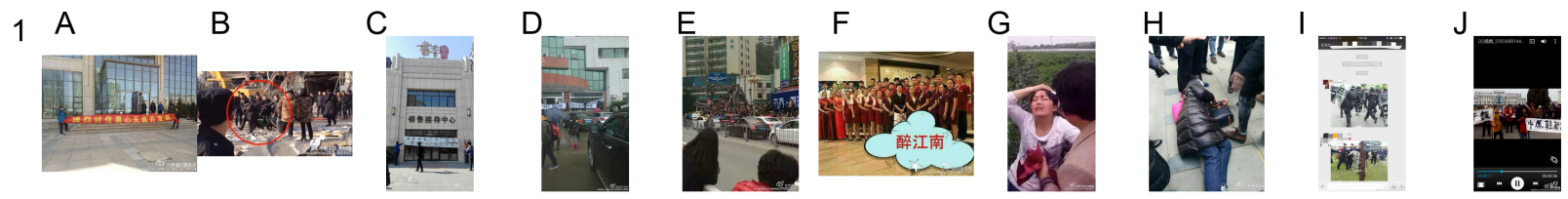

2
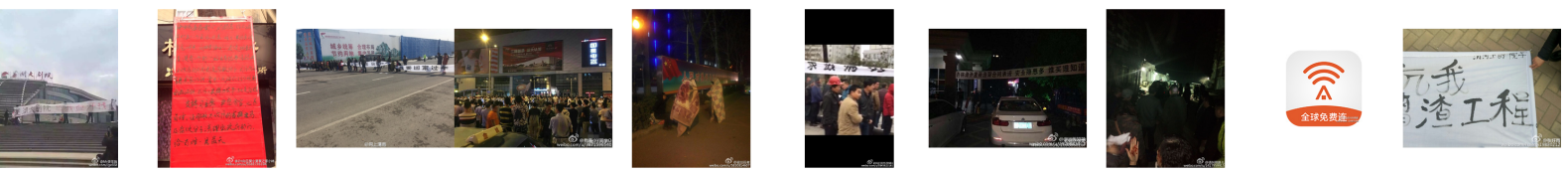

3
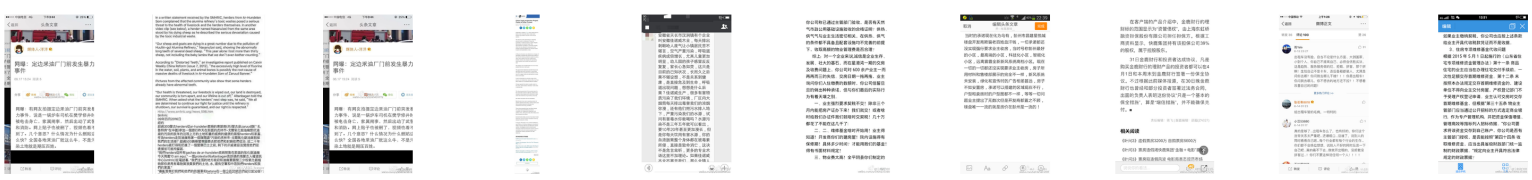

4
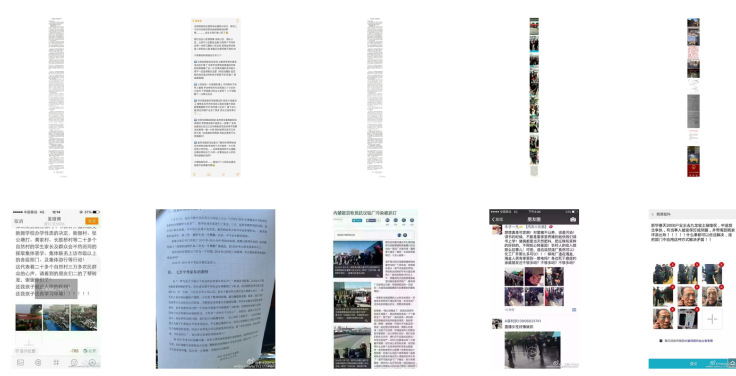

6
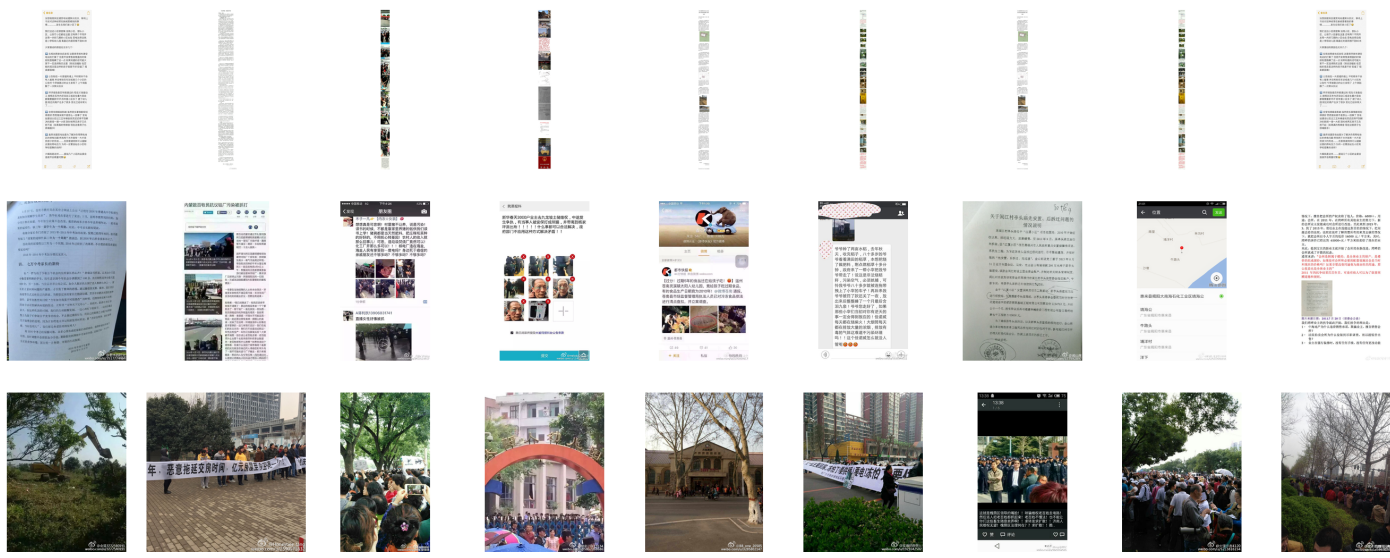

Figure 4: Method 1, Bag-of-Visual-Words model. 

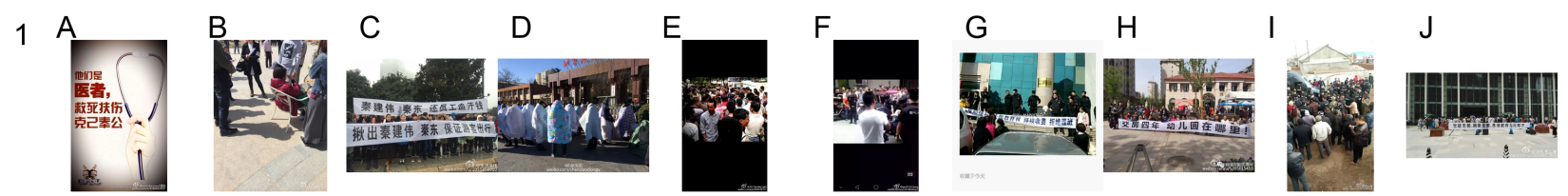

2
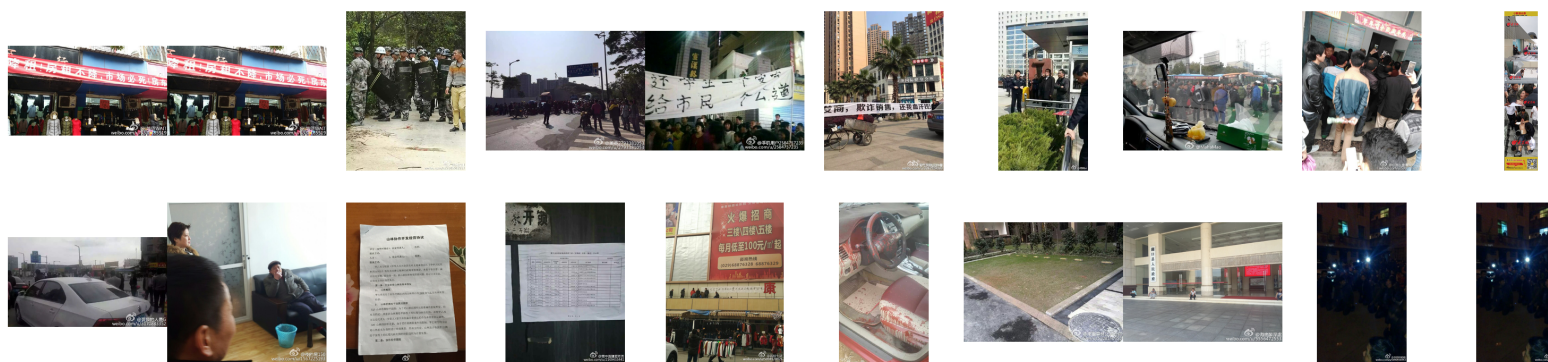

4
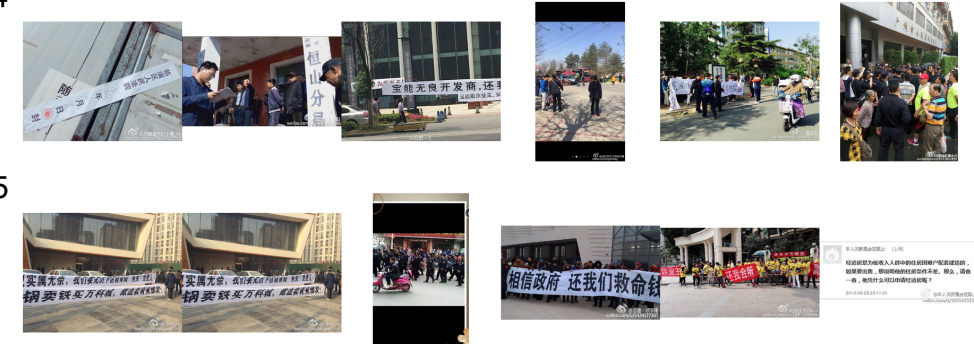

6
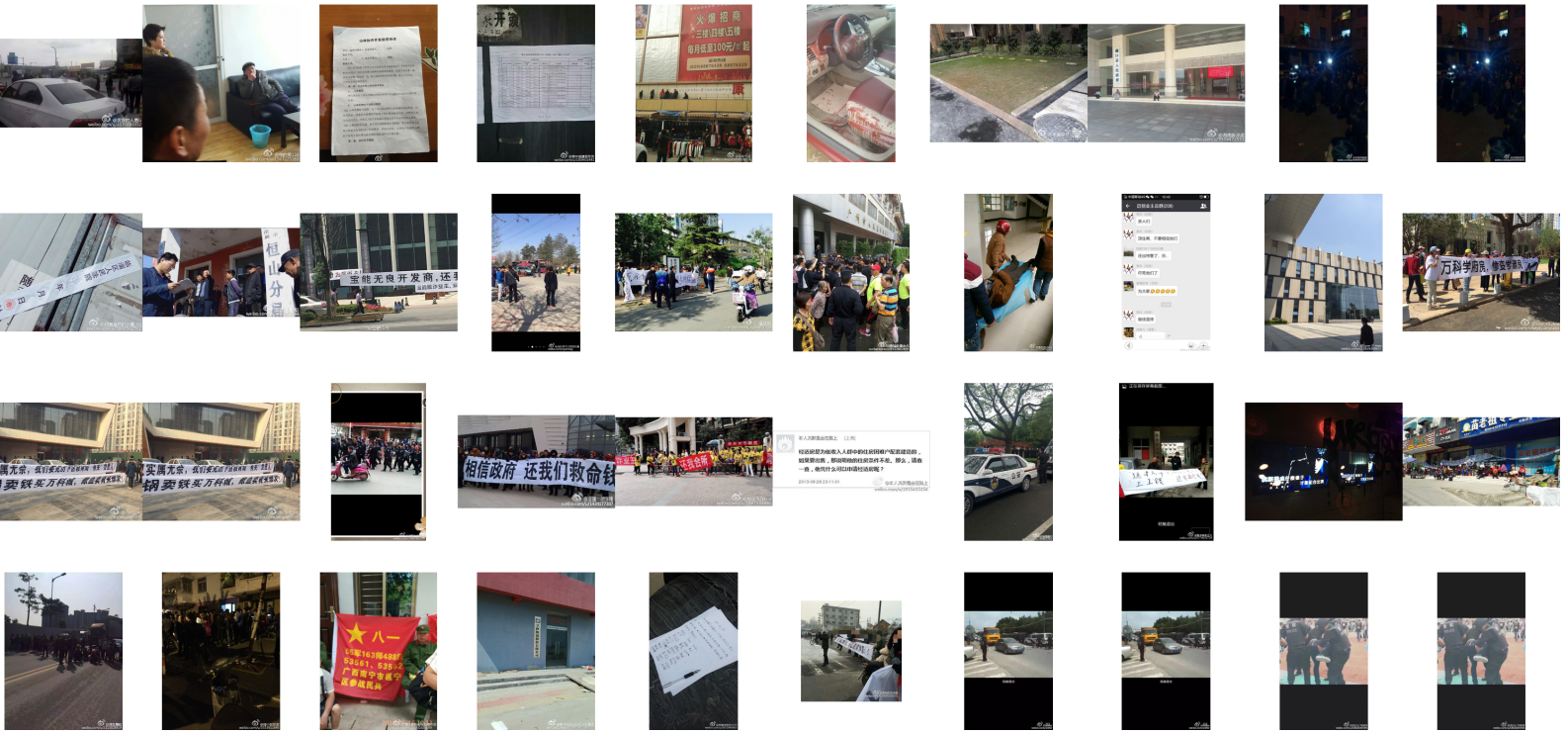

Figure 5: Method 2, self-supervised learning of image representations (Caron et al., 2018). 

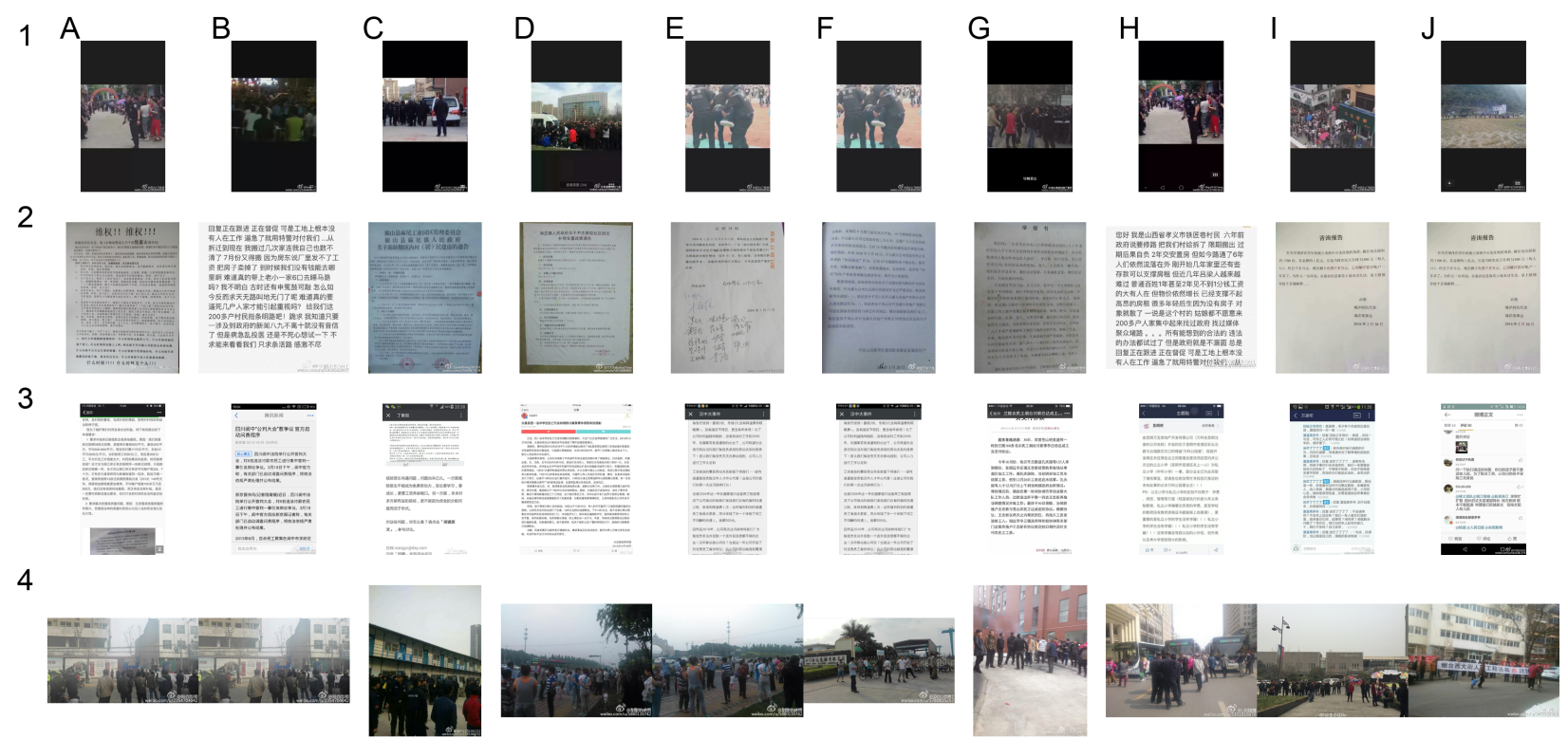

5
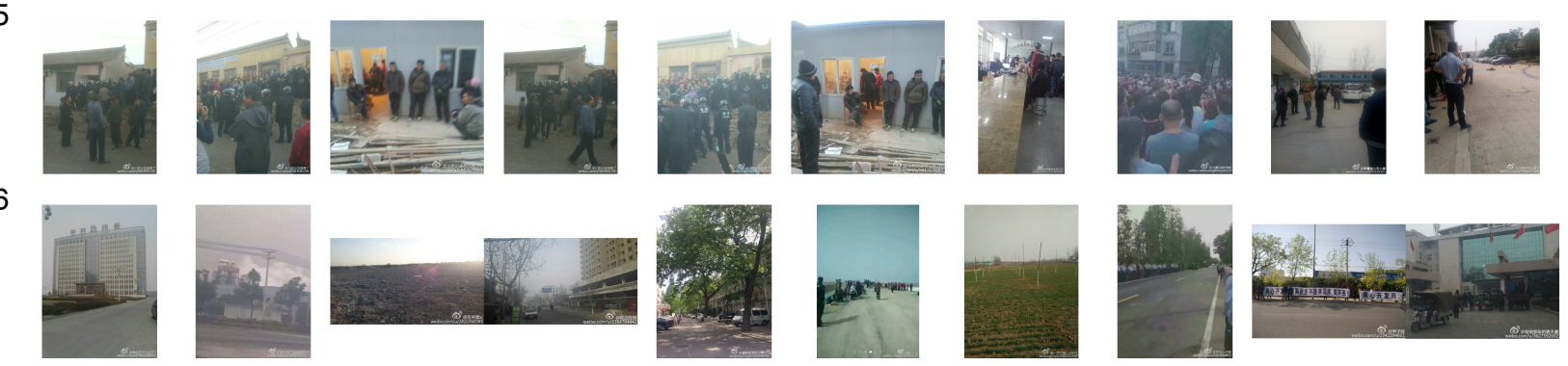

Figure 6: Method 3, transfer learning using supervised pretrained models. 


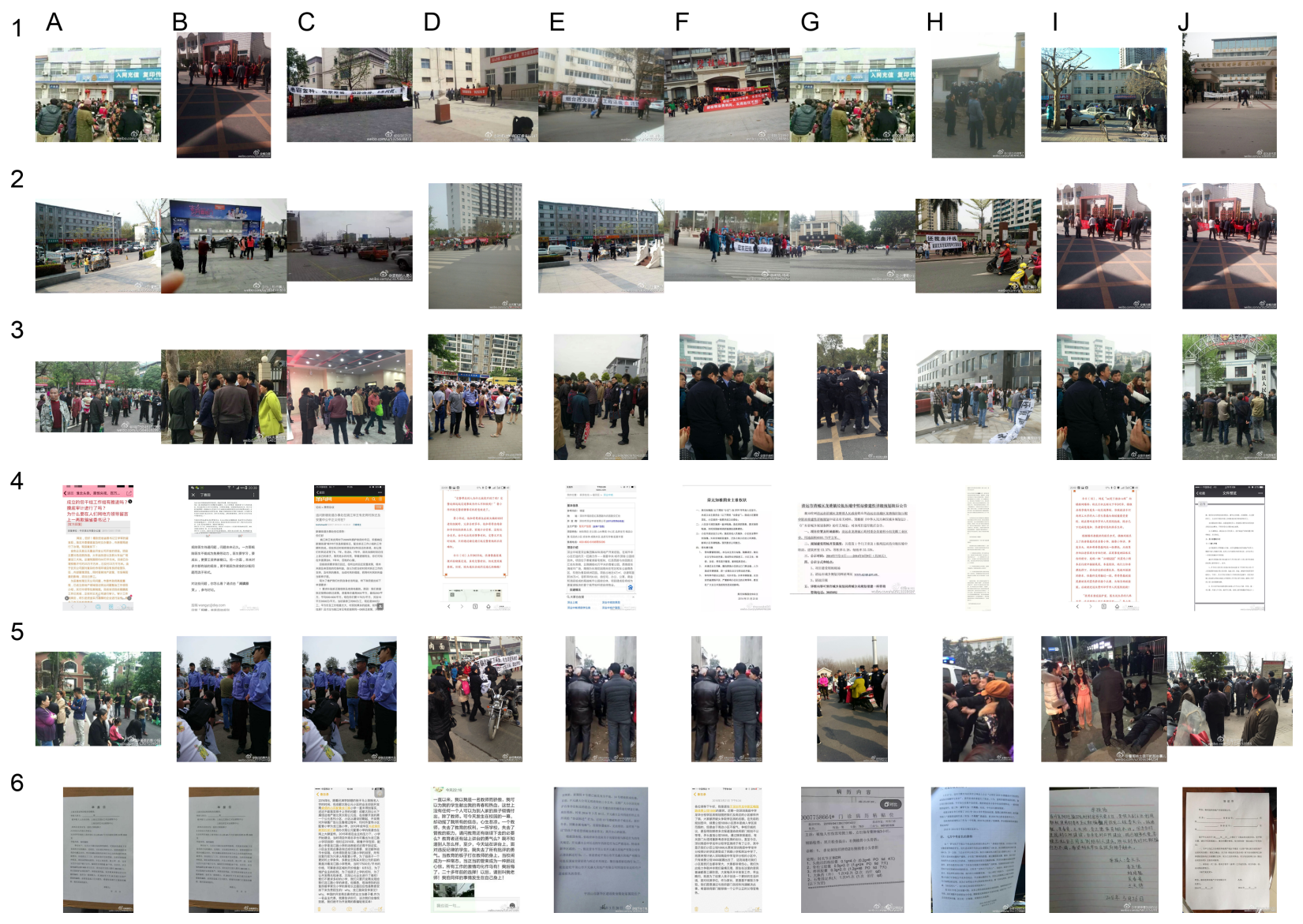

Figure 7: Method 3, transfer learning using self-supervised pretrained models. 


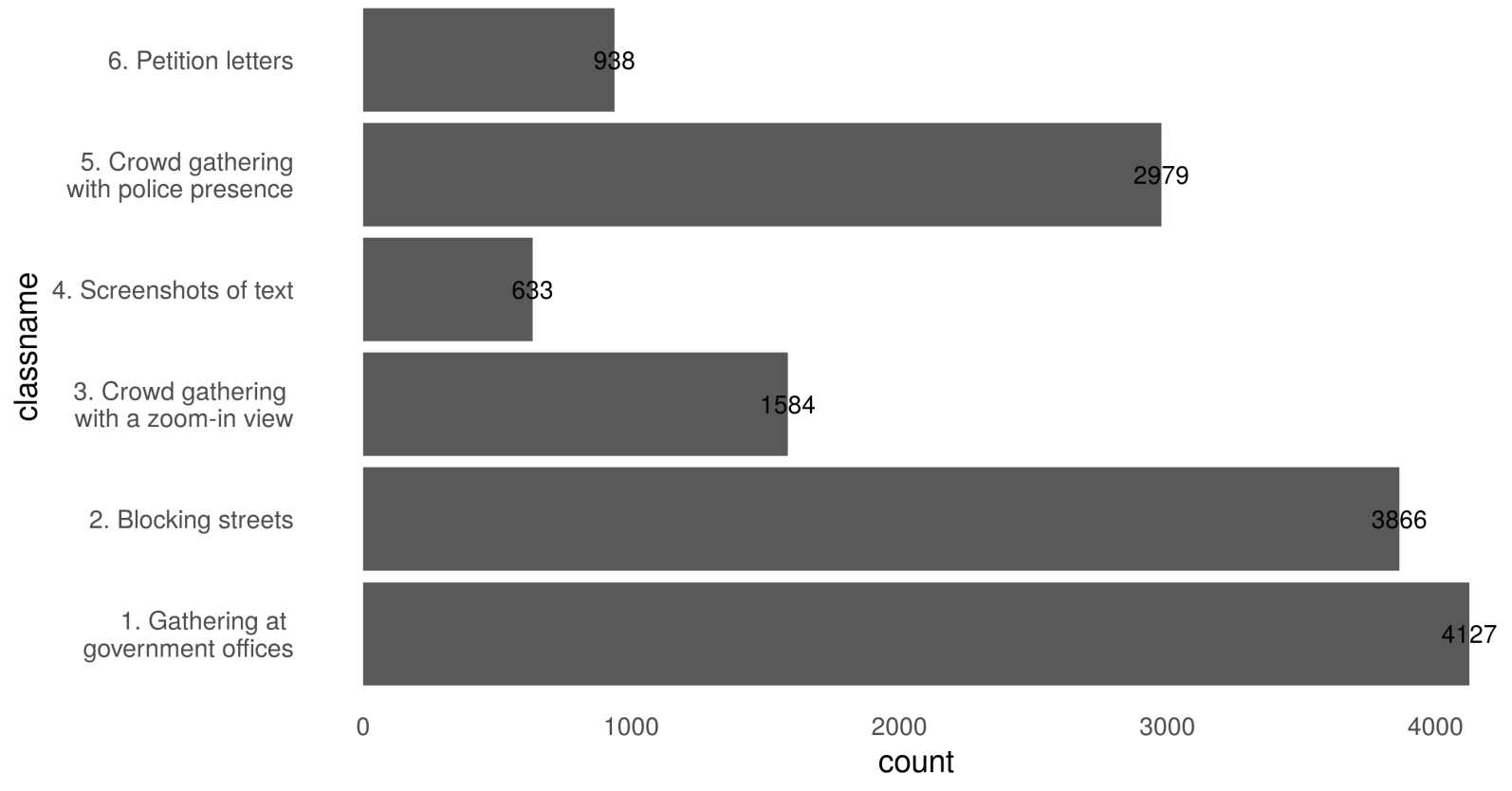

Figure 8: Number of images in each class. 


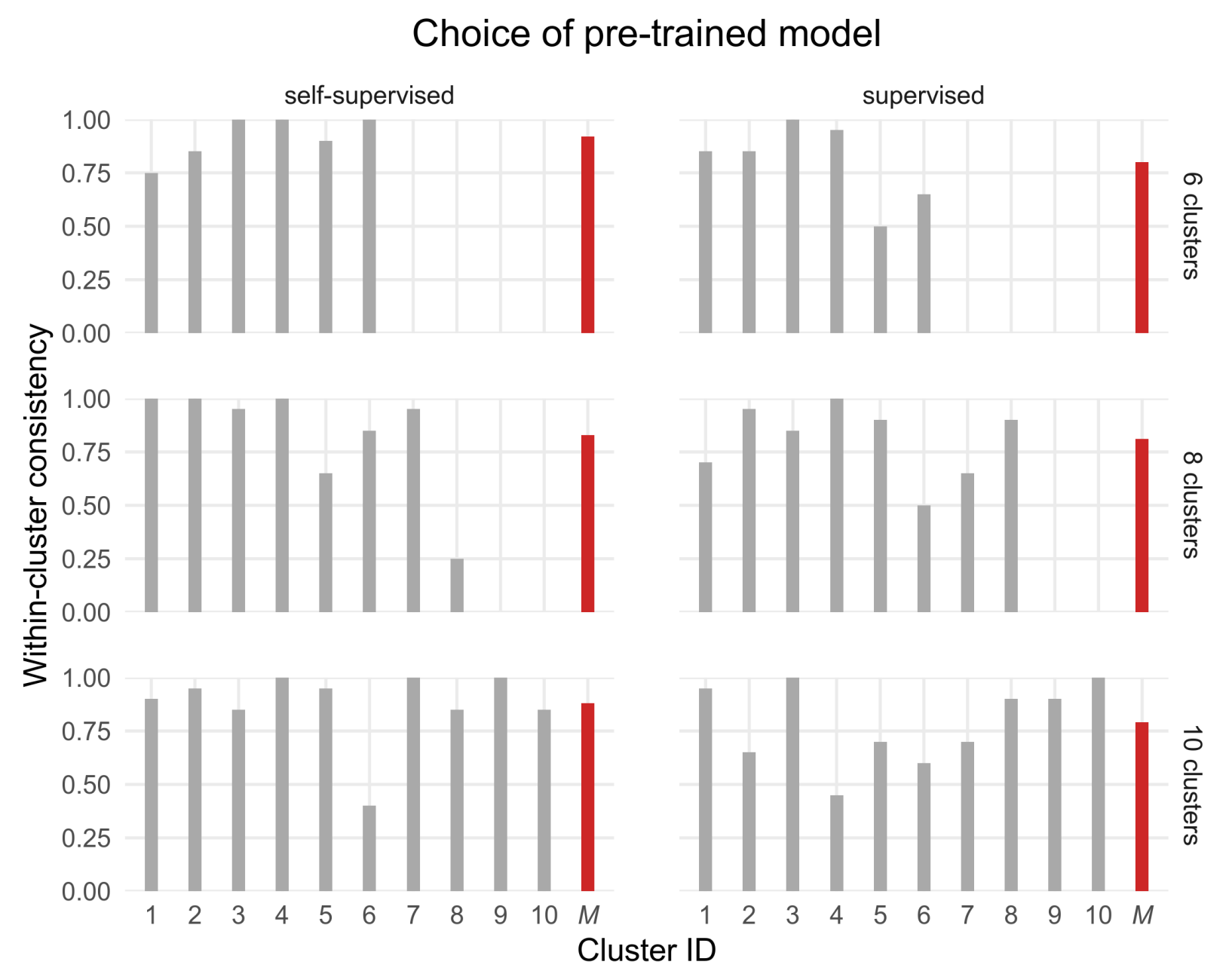

Figure 9: Percentage of images in each cluster that belong to the most common theme in that cluster, for Chinese Protest Dataset. Average percentage $(M)$ is highlighted in red. Six clustering solutions are shown, varying by the number of clusters and the pretrained models. 


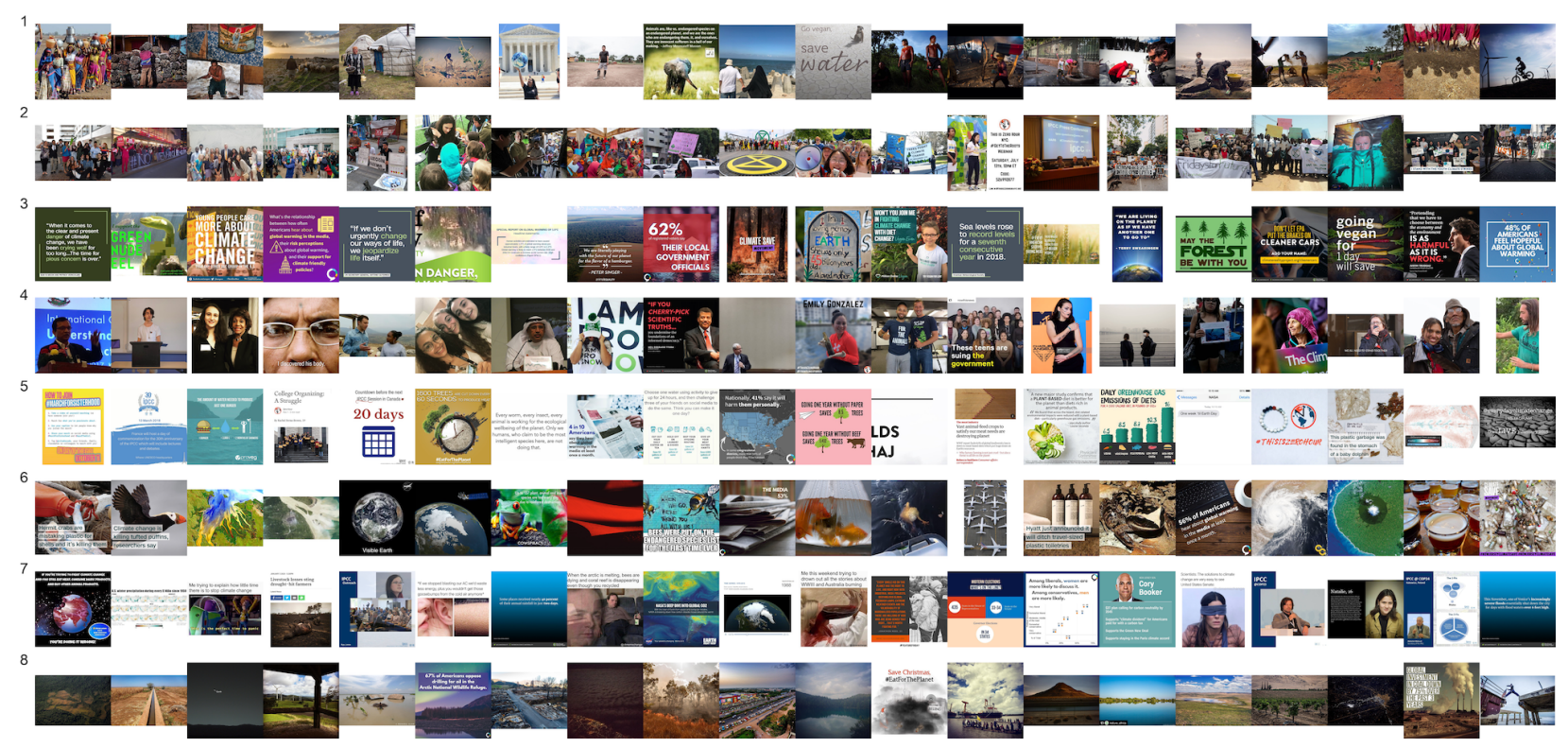

Figure 10: Eight-cluster solution based on features extracted from a pretrained model trained on ImageNet dataset. 


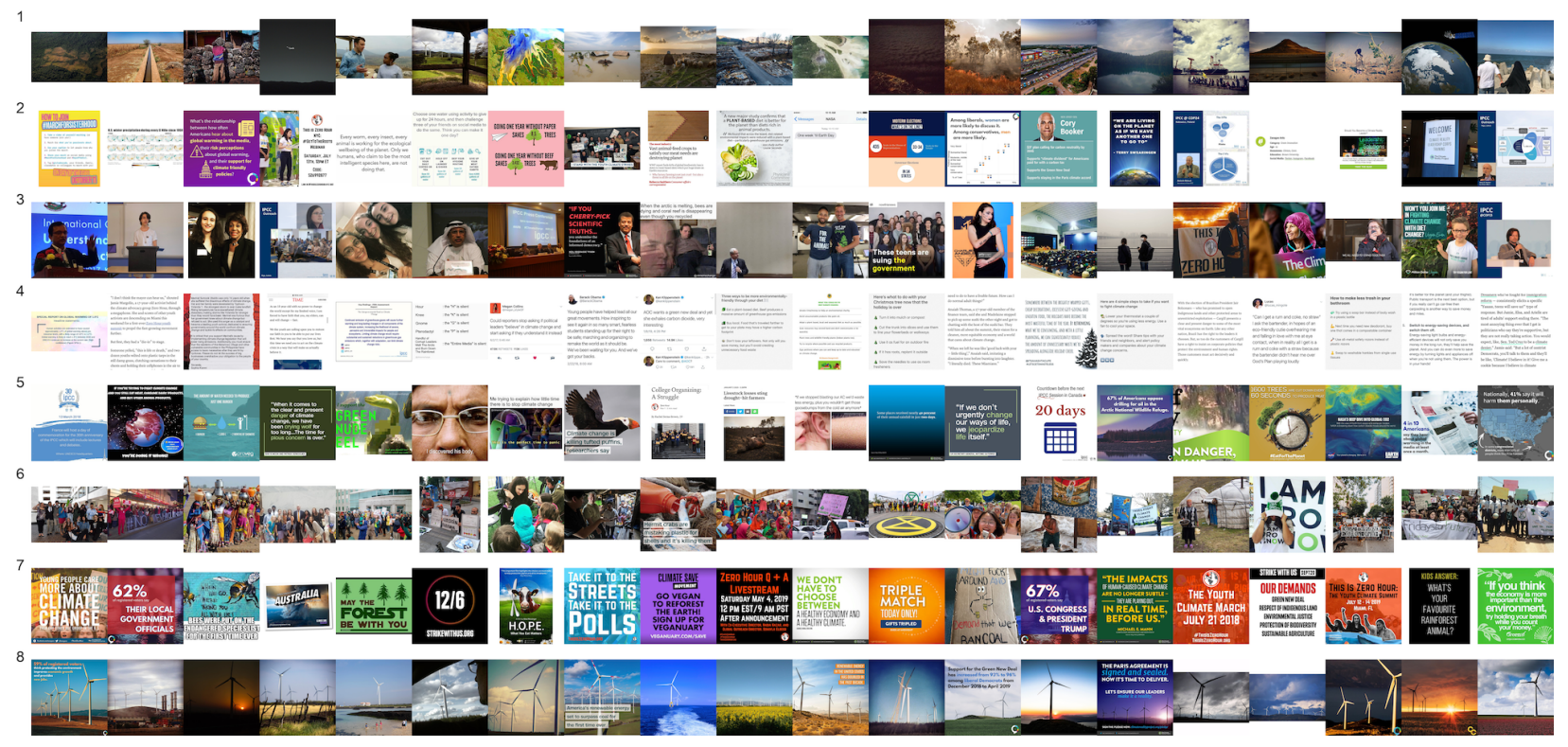

Figure 11: Eight-cluster solution based on features extracted from a pretrained model trained on the Places365 dataset. 


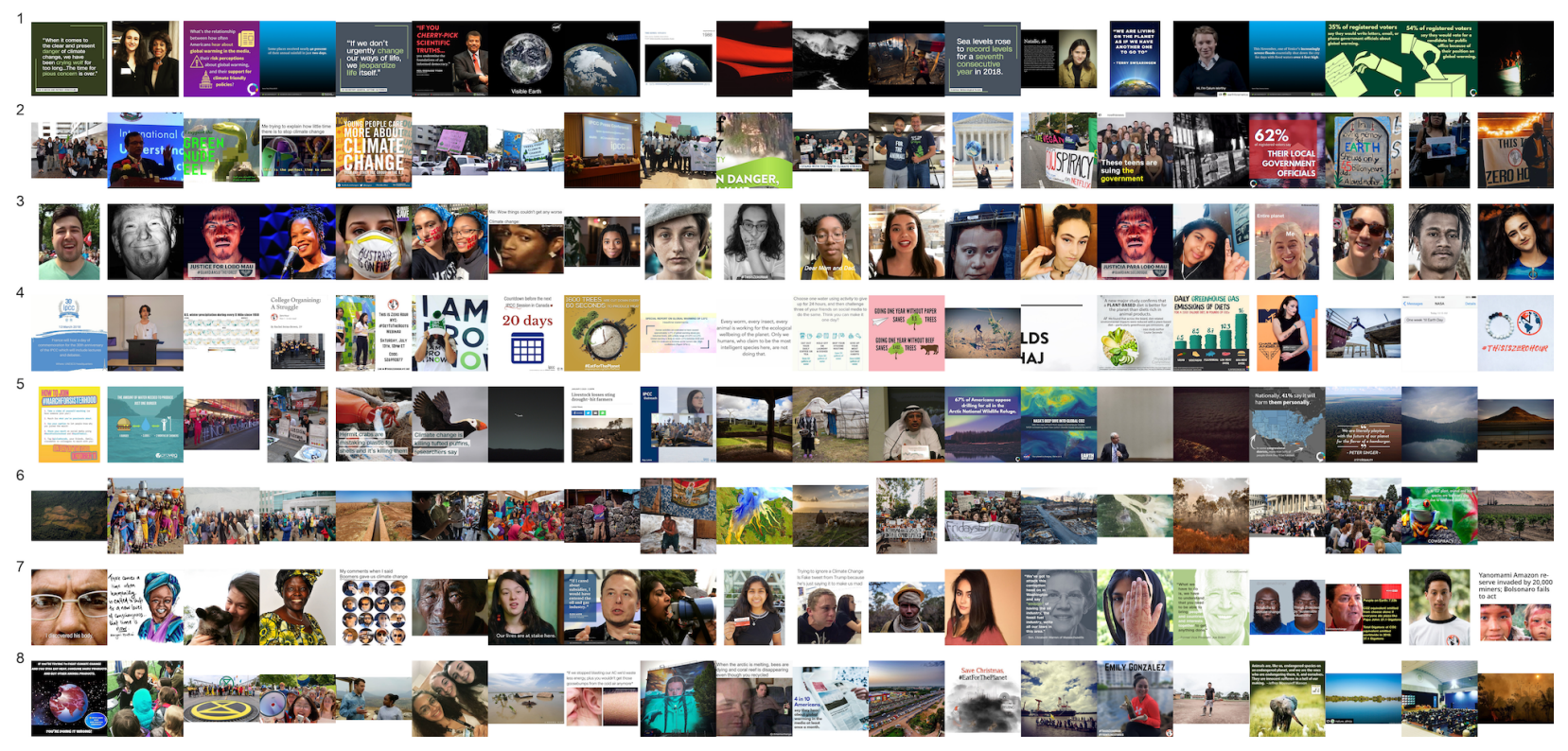

Figure 12: Eight-cluster solution based on features extracted from a pretrained model trained on the VGGFace dataset. 


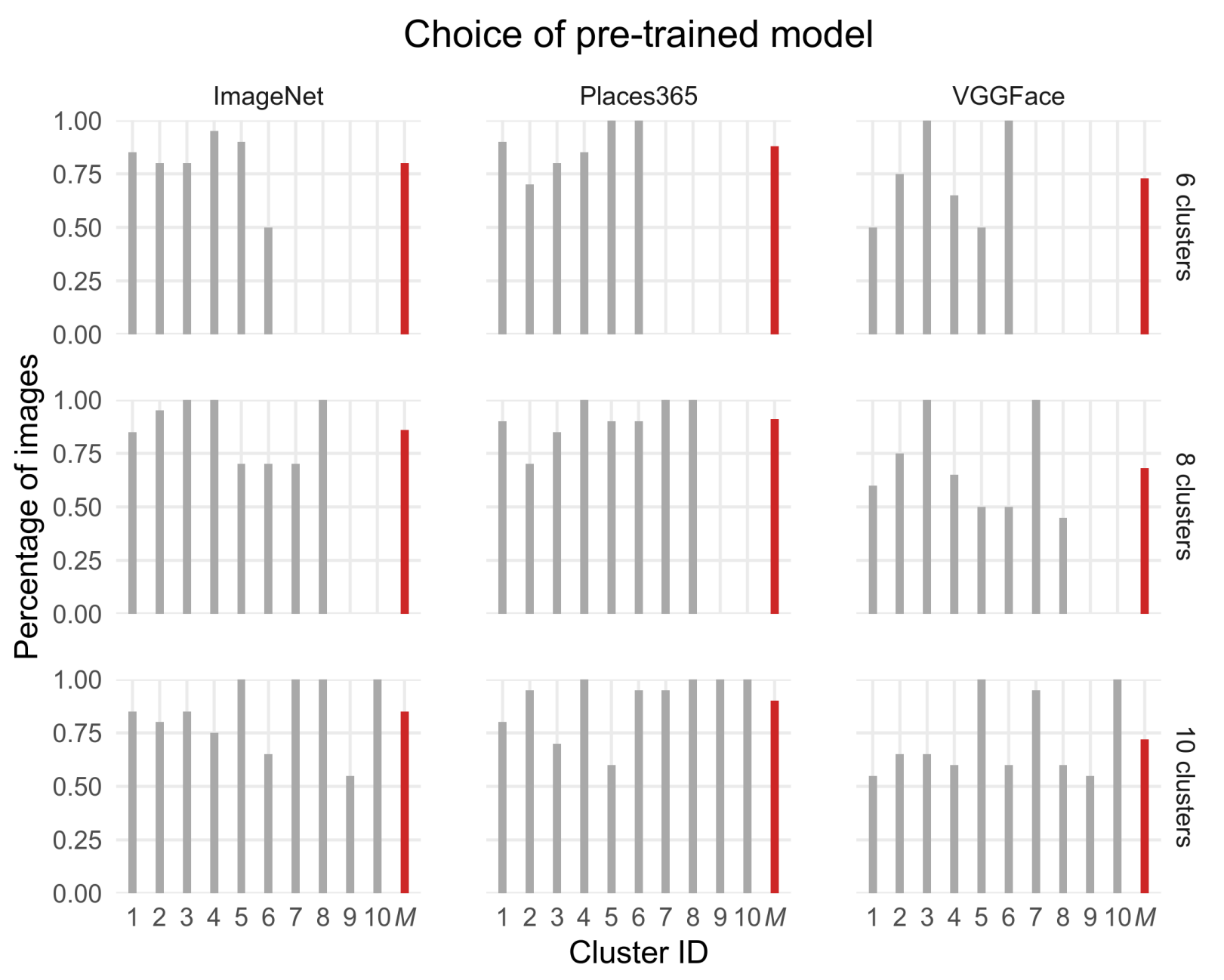

Figure 13: Percentage of images that belong to the most common theme in each cluster, for climate change dataset. Average percentage $(M)$ is highlighted in red. Nine clustering solutions are shown, varying by the number of clusters and pretrained models. 


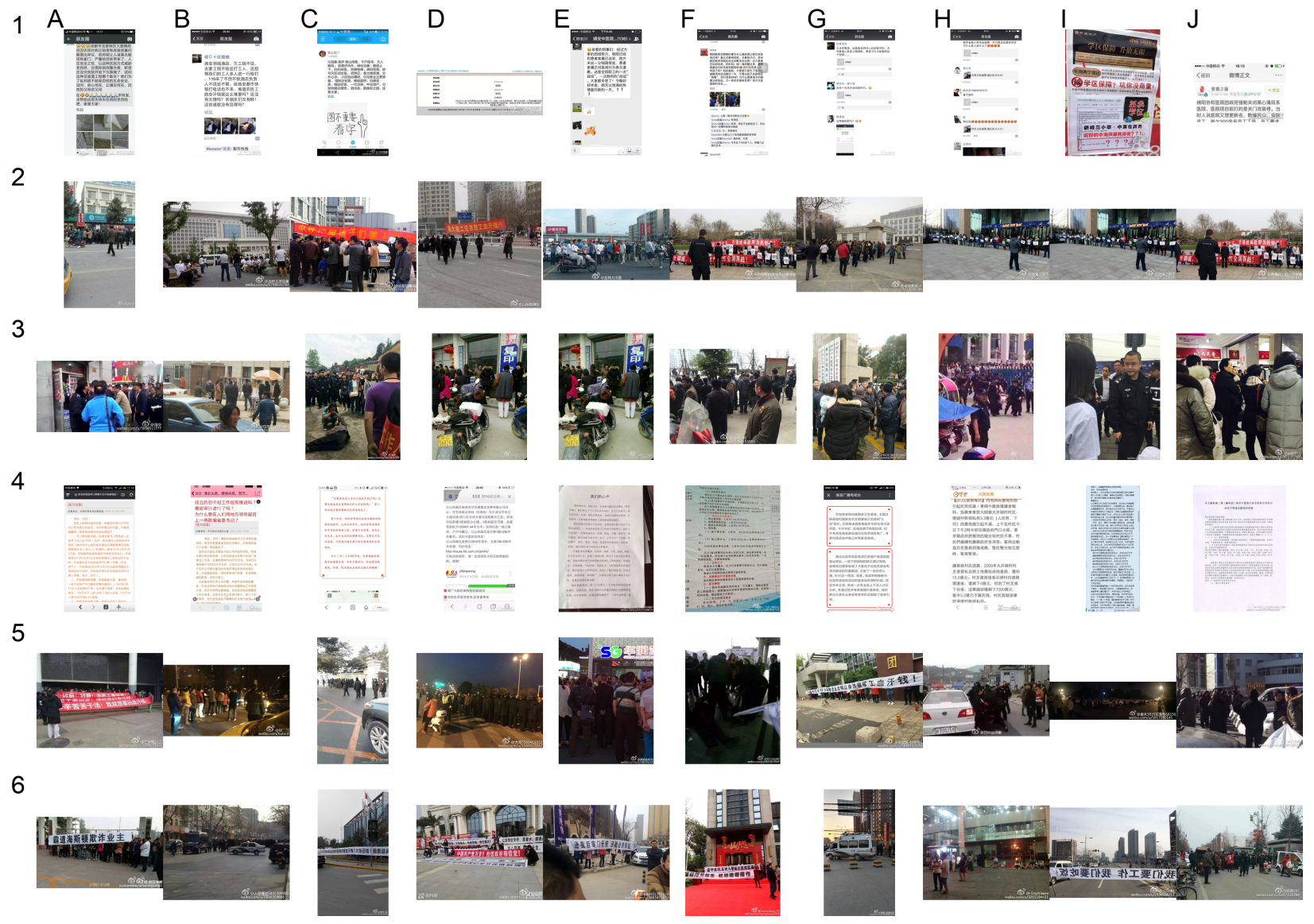

Figure 14: Method 3, transfer learning using self-supervised pretrained models (AlexNet as the architecture), on Protest Dataset 


\section{A Python code to perform transfer learning to obtain low-dimensional vectors}

Below is the code to extract low-dimensional vectors of images using the supervised pretrained model using standard VGGNet trained on ImageNet dataset. The extracted low-dimensional vectors are saved to disk, and scholars can freely choose any implementation of clustering algorithms (e.g., kmeans function in R or Sklearn package in Python).

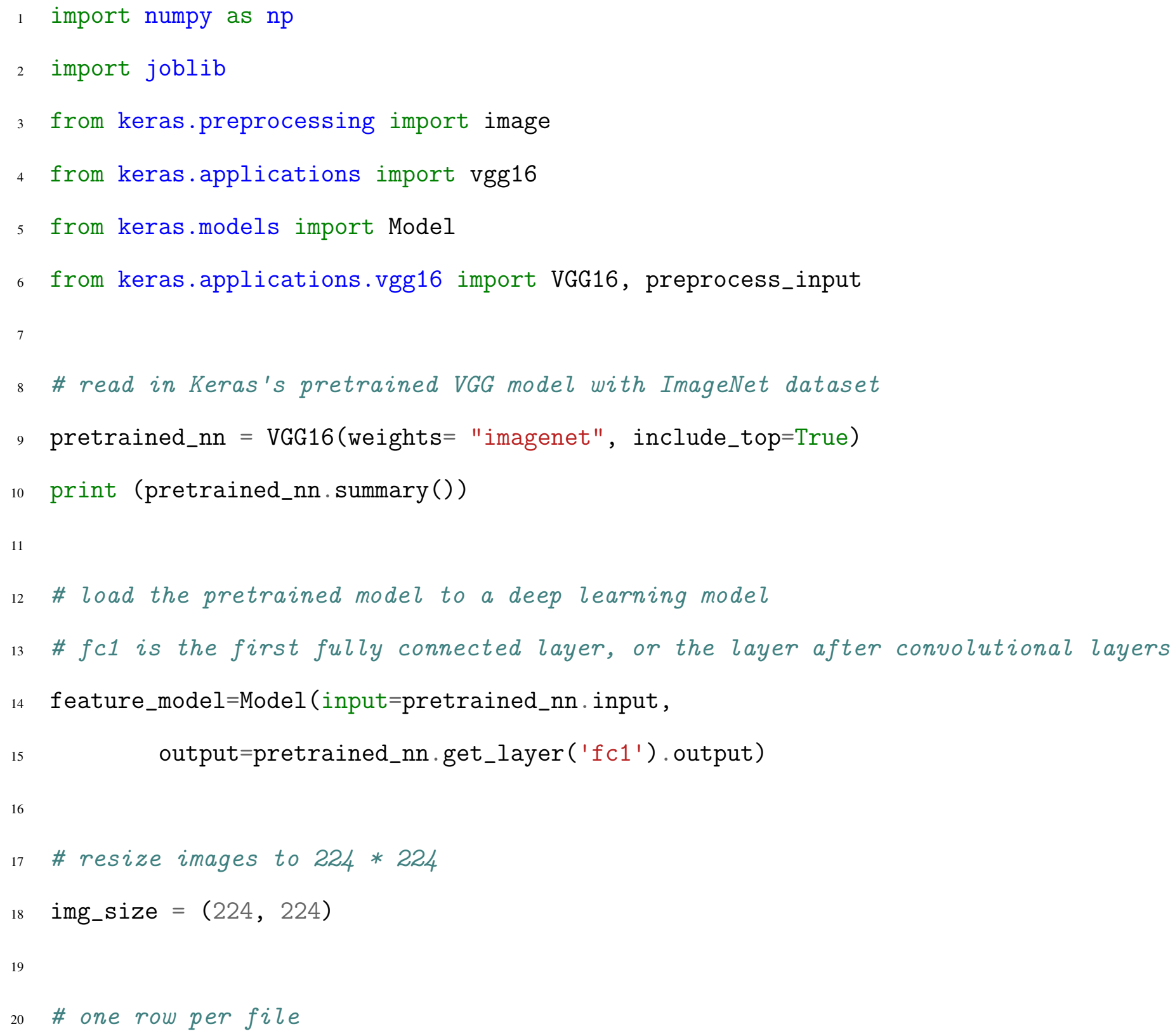




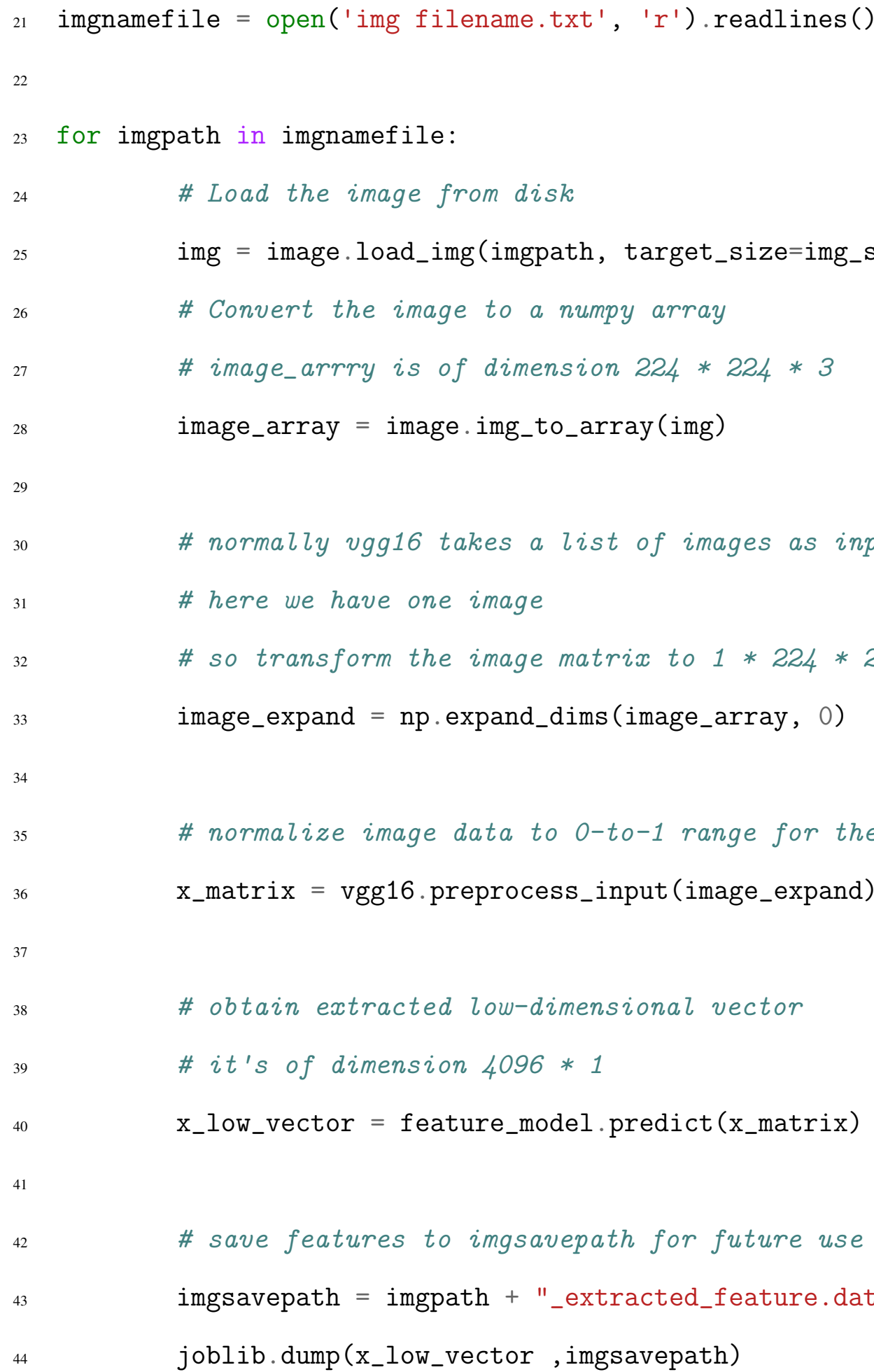

24

25

\section{B Different clustering algorithms}




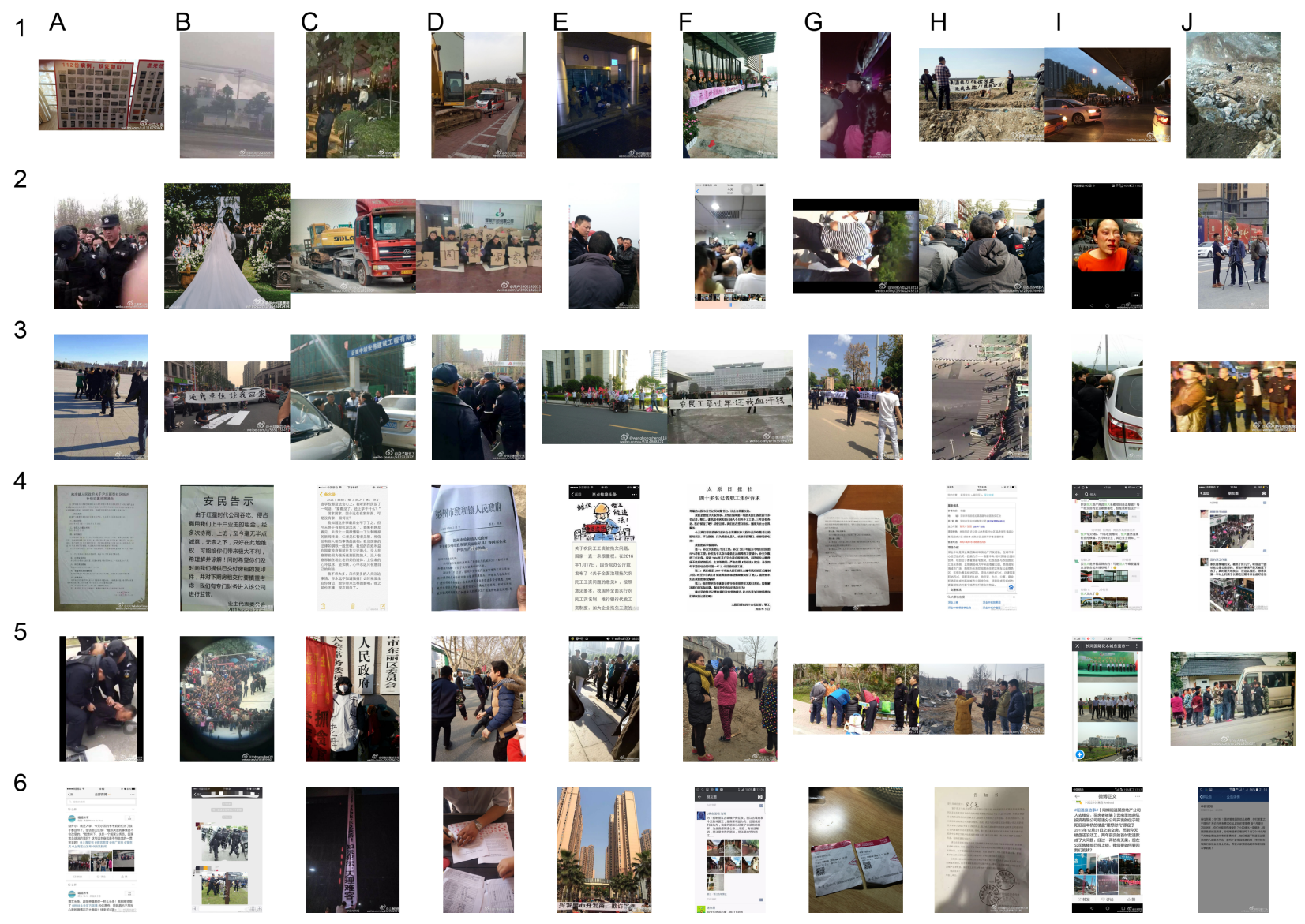

Figure B1: Transfer learning using self-supervised model, using agglomerative hierarchical clustering. Results are similar to Figure 7 where other things are equal but clustering algorithm is k-means. 


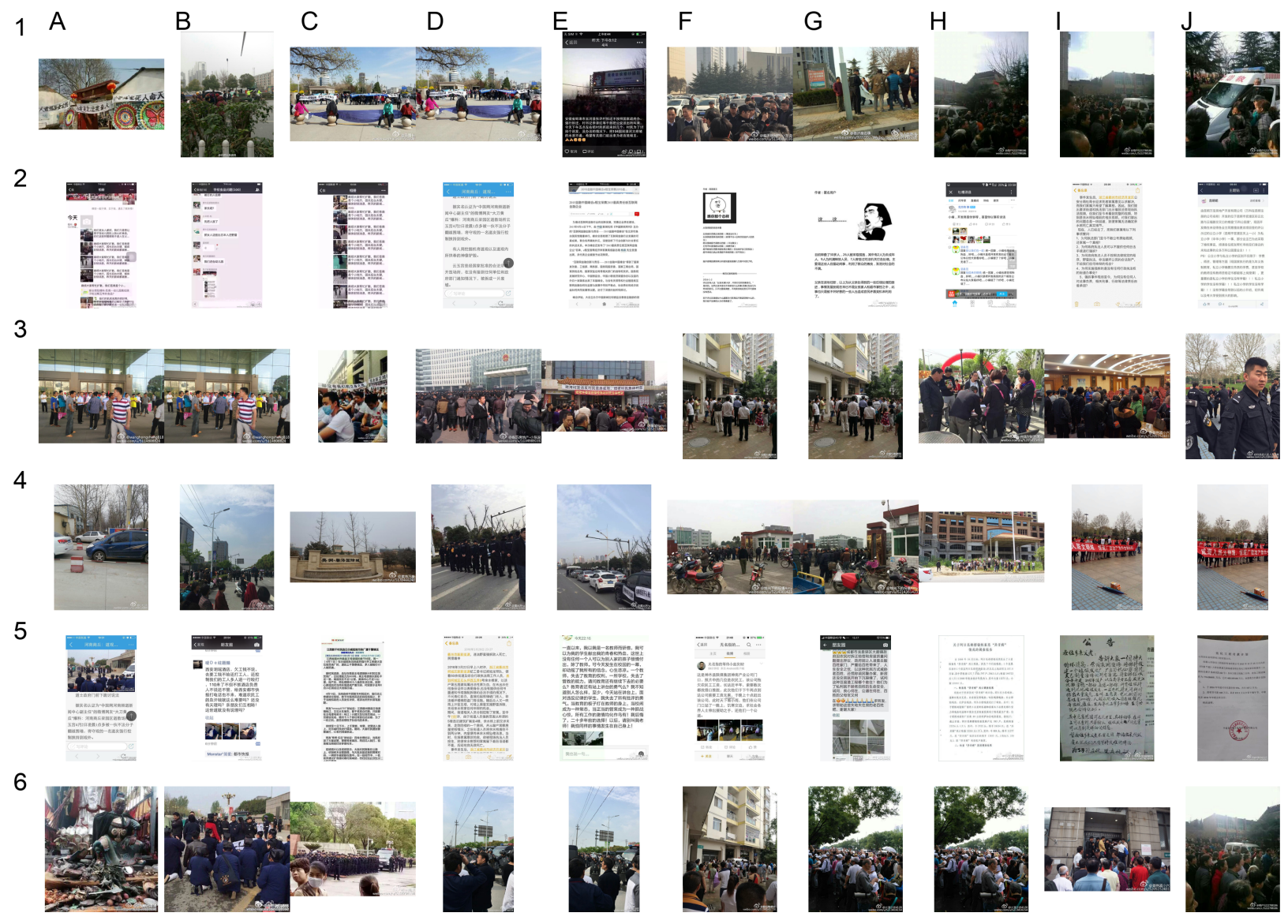

Figure B2: Transfer learning using self-supervised model, using Gaussian Mixture Model. Results are similar to Figure 7 where other things are equal but clustering algorithm is k-means. 